\title{
An Adaptive Threshold Framework for Event Detection Using HMM-Based Life Profiles
}

\author{
CHIEN CHIN CHEN \\ National Taiwan University \\ MENG CHANG CHEN \\ Academia Sinica \\ and \\ MING-SYAN CHEN \\ National Taiwan University
}

When an event occurs, it attracts attention of information sources to publish related documents along its lifespan. The task of event detection is to automatically identify events and their related documents from a document stream, which is a set of chronologically ordered documents collected from various information sources. Generally, each event has a distinct activeness development so that its status changes continuously during its lifespan. When an event is active, there are a lot of related documents from various information sources. In contrast when it is inactive, there are very few documents, but they are focused. Previous works on event detection did not consider the characteristics of the event's activeness, and used rigid thresholds for event detection. We propose a concept called life profile, modeled by a hidden Markov model, to model the activeness trends of events. In addition, a general event detection framework, LIPED, which utilizes the learned life profiles and the burst-and-diverse characteristic to adjust the event detection thresholds adaptively, can be incorporated into existing event detection methods. Based on the official TDT corpus and contest rules, the evaluation results show that existing detection methods that incorporate LIPED achieve better performance in the cost and F1 metrics, than without.

Categories and Subject Descriptors: H.3.3 [Information Storage and Retrieval]: Information Search and Retrieval—Clustering; H2.8 [Database Management]: Database Applications—Data mining; G.3 [Probability and Statistics]: Time series analysis

General Terms: Algorithm, Design, Experimentation

This research was supported in part by NSC 96-2218-E-002-040 and NSC 96-3113-H-001-012 from the National Science Council, Republic of China.

Authors' addresses: C. C. Chen, Department of Information Management, National Taiwan University, NO.1, Sec. 4, Roosevelt Road, Taipei City 106, Taiwan; email: paton@im.ntu.edu.tw; M. C. Chen, Institute of Information Science, Academia Sinica, 128, Sec. 2, Academic Rd., Nankang 115, Taiwan; email: mcc@iis.sinica.edu.tw; M.-S. Chen, Department of Electrical Engineering, National Taiwan University, No.1, Sec. 4, Roosevelt Road, Taipei City 106, Taiwan; email: mschen@cc.ee.ntu. edu.tw.

Permission to make digital or hard copies of part or all of this work for personal or classroom use is granted without fee provided that copies are not made or distributed for profit or commercial advantage and that copies show this notice on the first page or initial screen of a display along with the full citation. Copyrights for components of this work owned by others than ACM must be honored. Abstracting with credit is permitted. To copy otherwise, to republish, to post on servers, to redistribute to lists, or to use any component of this work in other works requires prior specific permission and/or a fee. Permissions may be requested from Publications Dept., ACM, Inc., 2 Penn Plaza, Suite 701, New York, NY 10121-0701 USA, fax +1 (212) 869-0481, or permissions@acm.org. (C) 2009 ACM 1046-8188/2009/02-ART9 \$5.00 DOI 10.1145/1462198.1462201 http://doi.acm.org/ $10.1145 / 1462198.1462201$ 
Additional Key Words and Phrases: Event detection, topic detection, TDT, life profiles, hidden Markov models, clustering

ACM Reference Format:

Chen, C. C., Chen, M. C., Chen, M.-S. 2009. An adaptive threshold framework for event detection using HMM-based life profiles. ACM Trans. Inform. Syst. 27, 2, Article 9 (February 2009), 35 pages. DOI $=10.1145 / 1462198.1462201$ http://doi.acm.org/10.1145/1462198.1462201

\section{INTRODUCTION}

Publishing activities on the Internet are now so prevalent that when an event occurs, autonomous reporters (called information sources in this paper) publish related documents in many forms, such as news reports, comments, and criticisms, over the event's lifespan. A document stream is composed of documents from information sources sequenced in chronological order. The task of event detection is to identify events and their related documents automatically from a document stream to help users understand events easily.

An event has various activeness development stages that affect the number of related documents. When an event is active, many related documents about various aspects of it are published, but when inactive, very few related documents are published. In addition to the dynamic activeness, the lifespan of events can be very different. A minor event, such as a car accident, might only appear for one or two days, while a major event, such as a war, could remain active for months. However, because Internet publishing activities are uncoordinated and there is no quality control, the content of documents related to an event may be imprecise or diverse, and may not be published in chronological order. These concerns make event detection an open and challenging research issue.

In recent years, the DARPA-sponsored Topic Detection and Tracking (TDT) project ${ }^{1}$ has promoted event detection research. The project defines an event as "something that occurs at some place and time associated with specific actions" and divides event detection tasks into online and offline processes. In the latter, also known as retrospective event detection, all documents are ready for processing; hence, traditional clustering methods like hierarchical agglomerative clustering (HAC) [Salton 1989] can be used to partition documents into coherent sets of documents efficiently [Yang et al. 1998]. However, in the online process, whereby documents are continuously and chronologically generated, traditional methods perform poorly, as their clustering performance relies on the availability of complete sets of documents. To meet the rapid increasing of online document streams, methods [Allan et al. 1998a; Chen et al. 2003; Makkonen et al. 2004; Yang et al. 1998] based on incremental clustering algorithm [Rijsbergen 1979] have been proposed to cluster documents sequentially. In the methods, documents are processed one by one in chronological order and each is considered to be a member of a cluster (i.e., detected event) if the similarity between it and the cluster is above a predefined clustering threshold. When a similar cluster cannot be found, the document itself forms a new cluster.

\footnotetext{
${ }^{1}$ http://www.nist.gov/speech/tests/tdt/index.htm 


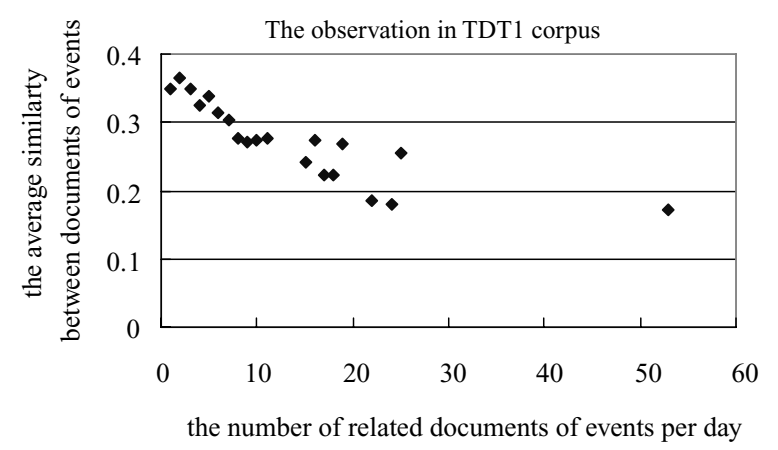

Fig. 1. Document similarity vs. event activeness.

One major problem with the incremental clustering algorithm is the threshold setting. Ideally, the clustering threshold should be adaptable to the status of an event to reflect the event's activeness developments. It is interesting to observe that the content of related documents of an event diversify when the event is active. Figure 1 illustrates such an observation about the DARPA TDT1 corpus [Allan et al. 1998b], in which news documents and events are labeled by human experts. For each labeled event, we collect the following statistics on a daily basis: (1) the number of related documents; and (2) the average similarity among the related documents. The documents are modeled by a vector space model (VSM) so that the similarity between two documents is the cosine similarity [Salton 1989]. In Figure 1, the $x$-coordinate is the number of documents generated per day, which can be regarded as an event's activeness indicator. Obviously, the more documents are published, the more active an event is. The $y$-coordinate shows the average similarity. Note a high average similarity value indicates that the content of an event is cohesive, while a low similarity value indicates the content is diversified. The trend in the figure shows that when an event is active, the similarity between related documents decreases. This is because, when an event becomes active, information sources publish documents about diverse aspects of it, and thus reduce the average similarity among the documents. For example, when an election campaign event is active, information sources report various aspects of it, such as details about the candidates and their families, the campaign agenda and strategies, poll results, criticisms and comments, and other tidbits. This "burst-and-diverse" phenomenon [Kleinberg 2002] of events corresponds to the dynamic behavior of a document stream. On the other hand, some events, such as a natural disaster in a remote country, do not attract much attention and have very few related documents, each of which may only have a lifespan of one or two days. For such events, documents tend to be highly focused and may be very similar. Based on the above observations, we believe that the performance of existing online event detection algorithms could be enhanced if an event's activeness status is known or predictable. When an event is active, using a lower clustering threshold would allow the inclusion of related, but diverse, documents without a severe reduction in the clustering precision. Then, when the event becomes inactive, raising the threshold would cause irrelevant documents to be rejected. 
In this article, we propose the concept of life profiles, which utilizes hidden Markov models (HMMs) [Markov 1913] to model an event's activeness development. Each life profile has a distinct characteristic of activeness development that can be learned from a set of events with similar patterns of activeness development. We also propose an adaptive event detection framework called LIPED (LIfe Profile-based Event Detection) that can be incorporated with existing event detection algorithms to improve their performances. During the event detection process of the framework, a detected event is dynamically associated with an appropriate life profile, which together with the event's activeness status, is used to determine a suitable clustering threshold. Our experiments show that LIPED improves the performance of existing well-known event detection approaches substantially.

The remainder of the article is organized as follows. In Section 2, we review prior works on event detection and status modeling of stream data. Life profile modeling and training are presented in Section 3. Section 4 describes the LIPED framework, and its performance is evaluated in Section 5. Finally, in Section 6, we present our conclusions.

\section{RELATED WORKS}

\subsection{Event Detection}

Event detection and traditional text clustering are similar in that both partition documents into coherent clusters. However, the temporal property of events makes their detection a more difficult and challenging research issue [Allan et al. 1998b]. Two popular representations of clusters are used in event detection algorithms: centroid-based and non-centroid-based approaches. In the centroid-based approach, an event is represented by a centroid aggregated from the contents of related documents of the cluster using a Rocchio-like formula [Rocchio 1971]. In the non-centroid-based approach, an event is described as a set of related documents, and the similarity between a document and an event is taken as the similarity between the document and the most similar document in the event. The time window method of [Yang et al. 1998] is a non-centroid based approach that represents an event as a collection of documents within a recent time window. The time window specifies the number of previous documents to be compared when clustering incoming documents. Events that do not have related documents within the window are considered as finished events to avoid several nonconcurrent, context-similar events being clustered together. Allan's time-based threshold method [Allan et al. 1998a], which is also a noncentroid-based approach, assumes that temporally close documents are likely to be associated with the same event. Hence, by adding a penalty value to the clustering threshold for temporally remote clusters, finished events are less likely to be associated with new documents. Chen et al. [2003] proposed the aging theory to control the lifespan of a centroid-based event. For an event, a real-number energy value is used to indicate its live status. The energy value increases as the event receives related documents, but declines with time. In addition, the larger number of related documents generated in a time unit for 
an event (which means the event is active), the longer the event will last. Li et al. [2005] modeled events in probabilistic manners. They proposed the use of a generative model to represent an event's content and timestamp information. Chou and Chen [2008] presented an incremental probabilistic latent semantic index algorithm to cluster online news documents sequentially. The authors showed that by modeling documents in latent semantics the acceptable threshold range for document clustering can be increased significantly. Zhang et al. [2004] applied a Dirichlet process prior to model the number of clusters in a document corpus. By estimating model parameters over historical datasets, the method can produce promising event detect results. Recently, natural language processing techniques have been applied to enhance event detection. Hatzivassiloglou et al. [2000] showed that linguistic features, that is, noun phrase and proper names, extracted from news documents, help to discriminate between context-similar events. Makkonen et al. [2004], moreover, built an ontology for the extracted location names to enhance the similarity calculation between documents.

\subsection{Status Modeling of Stream Data}

Modeling trends or the status of data in a stream environment has received a great deal of attention due to its many applications [Aggarwal 2003; Aizen et al. 2004; Donjerkovic and Ramakrishnan 2000; Ganti et al. 1999]. For example, on an E-commerce Web site, where transactions are generated continually, managers can analyze the purchase trends of products to adjust their business strategies. Aggarwal [2003] developed velocity density estimation to identify changes in evolving data streams. The method can be applied to GIS systems to monitor the trend of traffic in a certain area. The core of Aggarwal's method is based on kernel density estimation [Silverman 1986]. For an estimated spatial location, velocity density estimation calculates the difference between the forward and reverse density estimates within a time window. The density estimation utilizes kernel functions, such as the Gaussian distribution, as weighting functions to calculate the average change rate of data positions in the time window against a given location. The forward density estimate increases if data in the time window moves forward to the given location. On the other hand, if data moves outward from the location, the forward density estimate decreases. The definition of reverse density estimate is similar to the forward estimate, except that it uses a reversed time window. By subtracting these two density estimates, the velocity density estimation can determine whether recent data moves forward to or outward from the given location. The trend of the data can then be quantified precisely.

Aizen et al. [2004] utilized hidden Markov models (HMMs) [Markov 1913] to track the popularity of products on an E-commerce website. In this method, each state in the hidden Markov model represents a degree of popularity and the weights of arcs among states estimate the probability of popularity variations. Aizen's method considers each state as a Bernoulli random variable, which indicates the chance a user will buy a certain product. Therefore, products located in states with high Bernoulli parameters can be regarded as popular goods, and 
products in low-parameter states are less popular. Given the transaction log of an E-commerce web site, the popularity of a product can be identified by calculating its most likely state transitions. Even though Aizen's method can be used to measure a product's popularity, it violates an HMM constraint; that is, the sum of the weights of the outgoing arcs from a state must be equal to 1 . Since Aizen's method decides the weight of arcs by a heuristic method, the HMM constraint cannot always be satisfied. Another problem with Aizen's method is that it omits the timing factor; therefore, two sequential purchases in one hour are no different from two sequential purchases in one week. But, in reality, the former can be read as popular, while the later is unpopular. Finally, Aizen's method uses a single model to represent all types of popularity trends. However, according to Barlas and Kanar [1999], there are various styles of activeness development. Some developments burst in the beginning and gradually fade out, while others might start slowly and develop incrementally. Aizen's method would be more useful if it provided different models for different trends.

Summarizing these methods, we suggest a simple rule of thumb: the partial history of a stream of data can be used to predict the future behavior. Aggarwal uses a time window to predict the velocity density of a location and states in HMMs represent a kind of partial memory of recent occurrences. Our method is similar to Aizen's method in some degree. However, instead of using a single HMM to model various trends, we propose a framework that applies life profiles to model trends. Moreover, the proposed method takes time into account and its performance is more robust than Aizen's method. Finally, rather than use a heuristic to determine the arc weights, we propose a method to train the models so that the learned HMMs obey the HMM constraints fully.

\section{LIFE PROFILE MODELING}

Following the principle of burst-and-diverse characteristic in document streams, knowing the activeness status of events can help improve event detection performance. Life profiles describe distinct trends of an event's activeness; therefore, to know the activeness status of an event, we have to identify the event's life profile first. We now introduce the notations and definitions used in this paper. Let $O_{x}(t)=<o_{x, 1}, o_{x, 2}, \ldots, o_{x, t}>$ be the number of related documents published per day about an event $x$ from time 0 to $t$ (when without ambiguity, $O_{x}$ is used for $\left.O_{x}(t)\right)$ and let $L P=\left\{l p_{1}, l p_{2}, \ldots, l p_{L}\right\}$ be a set of life profiles. Then, given the event's previous behavior, $O_{x}$, the most likely life profile of the event would be:

$$
\hat{\imath}=\underset{1 \leq l \leq L}{\arg \max } P\left(l p_{l} \mid O_{x}\right) .
$$

By Bayes theorem [Ghahramani 2000], the above equation can be expanded as follows:

$$
\begin{aligned}
\hat{\imath} & =\underset{1 \leq l \leq L}{\arg \max } \frac{P\left(O_{x} \mid l p_{l}\right) P\left(l p_{l}\right)}{P\left(O_{x}\right)} \\
& =\underset{1 \leq l \leq L}{\arg \max } P\left(O_{x} \mid l p_{l}\right) P\left(l p_{l}\right) .
\end{aligned}
$$

ACM Transactions on Information Systems, Vol. 27, No. 2, Article 9, Publication date: February 2009. 


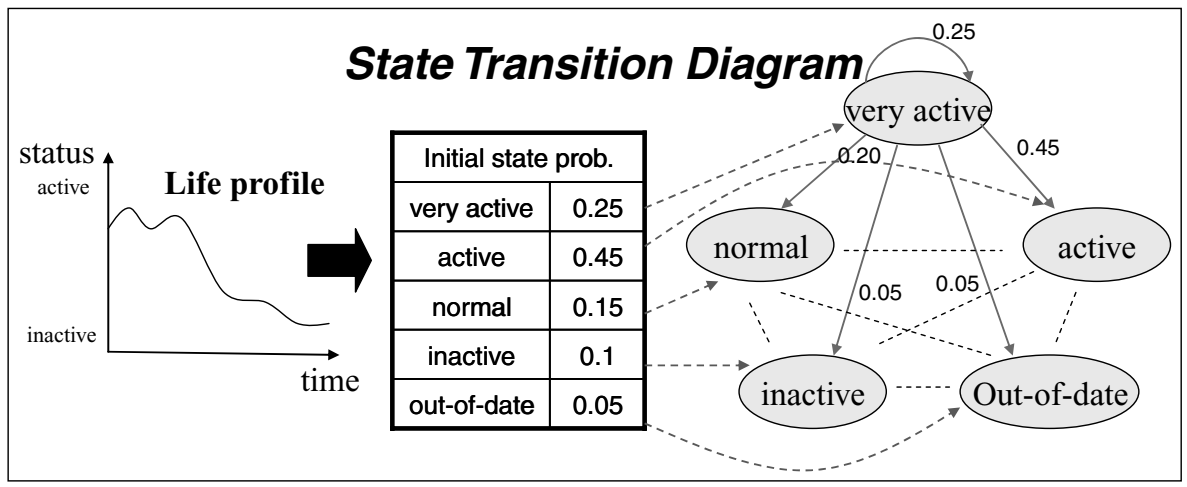

Fig. 2. Life profile and state transition diagram.

In other words, the most likely life profile of the event is the one with the maximum product of the likelihood $P\left(O_{x} \mid l p_{l}\right)$ and the prior probability $P\left(l p_{l}\right)$. If there is no feasible way to find the priors, it is reasonable to assume that the prior probabilities of every life profile are the same [Mitchell 1997]. Then, Equation (2) can be converted into the following form:

$$
\hat{l}=\underset{1 \leq l \leq L}{\arg \max } P\left(O_{x} \mid l p_{l}\right) .
$$

Now, the most likely life profile of the event is the one that has the maximum likelihood. To calculate the likelihood values, we have to model life profiles first.

A life profile can be modeled as a probabilistic state transition diagram. An example of such modeling is shown in Figure 2. In the model, the activeness of an event is represented by a set of states $S=\left\{\right.$ state $_{1}$, state $_{2}, \ldots$, state $\left._{N}\right\}$, where each state represents a degree of activeness. Additionally, the model comprises two kinds of probabilities: an initial state probability for each state, and a state transition probability for every pair of states. For every time slot, the transition of an event from its current state to the next state depends on the transition probability. The state transition probabilities are represented as an $N \times N$ matrix, $A$, in which an element $\alpha_{i, j}$ designates the probability of the transition from state $i$ to state $j$, and can be interpreted in terms of conditional probability, that is, $a_{i, j}=P\left(\right.$ state $_{j} \mid$ state $\left._{i}\right)$. The initial state probabilities are represented by a vector $\Pi$, where an element $\pi_{i}$ indicates the possibility that a life profile begins at state $i$. Note that, to follow the summation-one axiom of probability [Ghahramani 2000], the sum of the initial state probabilities and the sum of the outgoing transition probabilities of each state must be equal to 1 ; that is, $\sum_{i=1}^{N} \pi_{i}=1$ and $\sum_{j=1}^{N} a_{i, j}=1, \forall i$, where $\pi_{i} \geq 0, \forall i$ and $a_{i, j} \geq 0, \forall i, j$.

In reality, any state transition diagram is a Markov model, since it possesses two Markov properties: limited horizon and stationary [Rabiner 1989]. By modeling a life profile as a state transition diagram, the likelihood of Equation (3) can be obtained when the state transition sequence of the event is known. The value is then calculated by multiplying the weights of the traversed arcs and the initial state probability. However, in practice, the only fact we know about the event is its outcome sequence, that is, $O_{x}$. Therefore, the relationship between 
state transitions and the outcomes needs to be determined to obtain the likelihood value. We extend our life profile modeling from a Markov model to a hidden Markov model (HMM). Basically, one can regard HMMs and Markov models as the same, except that during a state transition, HMMs emit an outcome value. Therefore, HMMs have an additional set of probabilities: the probability of outcome values. With the outcome values and their probabilities, HMMs make the likelihood $P\left(O_{x} \mid l p_{l}\right)$ calculable. In this paper, we adopt the state-emission HMMs, where the outcome produced by a state transition depends solely on the end state. We use the symbol $B$ to represent the outcome symbol probabilities, and $b_{i, k}$ to denote the probability of emitting $k$ after entering state $i$. Formally, an HMM-based life profile $l p$ can be specified by a five-tuple $(S, K, \Pi, A, B)$, where $S$ is a set of states of an event, and $K$ is the domain of observed outcomes. $\Pi, A$, and $B$ are the probabilities of the initial states, the state transitions, and the outcome symbols, respectively. In the following subsections, we illustrate how these five components can be acquired from a training data set.

\subsection{Acquiring $K, S$, and $B$}

A set of observed outcomes $K$ is application-dependent. In this study, an outcome is the number of related documents published per day. Thus, $K$ is a set of natural numbers. The number of states in $S$ is specified by a framework parameter $N$. The larger $N$ is, the more representation power a life profile has. However, a large $N$ also increases the complexity of life profile training. Furthermore, the performance improvement may be negligible once the number of states is sufficiently large [Aizen et al. 2004; Kleinberg 2002]. After specifying the value of $N$, we construct a set $\mathrm{S}=\left\{\right.$ state $_{1}$, state $_{2}, \ldots$, state $\left._{N}\right\}$ for a life profile.

The outcome symbol probabilities $B$ are determined by the given training examples. A training example is a sequence of observed outcomes of an event, $O_{x}=<o_{x, 1}, o_{x, 2}, \ldots, O_{x,\left|O_{x}\right|}>$. The symbol $\left|O_{x}\right|$ indicates the lifespan of an event $x$. An element $o_{x, t}$ is the observed outcome at time slot $t$. From the training examples, we obtain the $\max$ and $\min$ statistics about the observed outcome. Then, a state $i$ is associated with a parameter $\omega_{i}$ by the following formula:

$$
\omega_{i}=\min +\Delta *(i-1)
$$

where $\Delta=\lceil(\max -\min ) / N\rceil$. We treat the emitted outcome of each state as a random variable with a sample space $K$, and the value $b_{i, k}$ is the corresponding probability in state $i$. Since the observed outcome of an event is a discrete value, a discrete probability distribution is employed. Each state may be thought of as a Poisson random variable. Then, the probability $b_{i, k}$ is calculated by the following formula:

$$
b_{i, k}=e^{-\omega_{i}\left(\omega_{i}\right)^{k}} / k !
$$

Note that the formula is similar to the probability mass function of a Poisson distribution by replacing the Poisson probability parameter $\lambda$ with $\omega_{i}$.

One important benefit of using probability distributions for observed outcomes is that the HMM constraint holds that is, for every state, the probability mass of all possible outcomes must be 1 . When the domain of an observed 
outcome is indefinite or infinite, such as the natural numbers of $[0, \infty)$, the constraint is very difficult to preserve without using a mathematically sound method, such as that proposed above. Another benefit of the proposed method is that it associates each state with real world phenomena, according to the applications and outcome distributions. For instance, when the observed outcome of an event is the number of related documents published per day, states with a high $\omega_{i}$ value can be regarded as active because events in such states will likely generate many related documents.

\subsection{Acquiring $A$ and $\prod$}

Let the set of observed outcome sequences $\left\{O_{1}, O_{2}, \ldots, O_{M}\right\}$ be the training events of a life profile $l p$. The acquired $A$ and $\Pi$ together with $S, K$, and $B$ are expected to maximize the following probability:

$$
P\left(\left\{O_{1}, O_{2}, \ldots, O_{M}\right\} \mid S, K, B, A, \Pi\right) .
$$

In other words, the desired $A$ and $\Pi$, together with the other model components, best interpret the training events. If the training events faithfully reflect the information about state transitions, the values of $A$ and $\Pi$ can be calculated by using maximum likelihood estimation. For example, if there are ten transitions in the training events departing from state $i$ and six of them move to state $j$, then the value of $a_{i, j}$ based on maximum likelihood estimation is $6 / 10$. Analogously, if one in four of the training events begins at state $i$, then the initial state probability $\pi_{i}$ based on the maximum likelihood estimation is $1 / 4$. However, in practice, the only fact we know about a training event is the observed outcome sequence; the corresponding state transitions are not explicitly represented. Thus, to obtain the values of $A$ and $\Pi$ from the implicit training events, we adopt the Forward-Backward algorithm [Baum et al. 1970], a type of EM algorithm [Dempster et al. 1977] frequently applied to estimate model parameters from incomplete data.

The training events are incomplete because the information about the state transitions is unobservable. Formally, let $O_{x}=\left\langle o_{x, 1}, o_{x, 2}, \ldots, O_{x,\left|O_{x}\right|}\right\rangle$, where $o_{x, i} \in K$, be the observed outcome sequence of a training event and $<s_{x, 1}, s_{x, 2}, \ldots, S_{x,\left|O_{x}\right|}>$, where $s_{x, i} \in S$, be the states that the event visits, that is, at time $t$, the event traverses from state $s_{x, t-1}$ to $s_{x, t}$, and emits an observed outcome $o_{x, t}$. Then the complete representation of a training event is $\left.O_{x}^{\prime}=\left(<o_{x, 1}, o_{x, 2}, \ldots, O_{x,\left|O_{x}\right|}\right\rangle,<s_{x, 1}, s_{x, 2}, \ldots, S_{x,\left|O_{x}\right|}>\right)$. Note that the lengths of training events may differ due to their distinct lifespans. Given the training events for a life profile, the Forward-Backward algorithm first randomly initializes the values of $A$ and $\Pi$, and iteratively executes the following two steps [Chen et al. 2005] until Equation 6 converges.

$-E$ step: estimate the unobservable state transitions of each training event using the current life profile.

$-M$ step: refine the life profile components $A$ and $\Pi$ by maximum likelihood estimation, given the estimated state transitions calculated in the previous step. 
The previous sections illustrate how a life profile can be obtained from training events. To sum up, we first calculate the max and $\min$ statistics from the training events. Next, we construct a state diagram for a life profile with $N$ states and treat each state as a random variable to obtain the outcome probabilities by Equation (5). Finally, the E-M steps are run iteratively to determine the initial state and the state transition probabilities. With each iteration of the $E$ and $M$ steps, the likelihood between a life profile and its training events rises. Even though this process may not find the optimal values of $A$ and $\Pi$, the re-estimation step guarantees that the profile components will converge to a local maximum [Rabiner 1989].

\section{LIPED}

In this section, we present the LIPED (LIfe Profile-based Event Detection) framework. A unique feature of the proposed framework is that the clustering threshold, used to decide whether a document is similar enough to be a member of a cluster, is adaptive to the activeness status of an event. LIPED can be easily incorporated into existing event detection systems to enhance their performance. For ease of illustration, the following sections present LIPED using an incremental clustering algorithm. We first describe the data models and then present the procedures for event detection, event status maintenance, and strategies for clustering thresholds.

\subsection{LIPED Data Models}

Documents and clusters are two basic data units in event detection. We represent each document as a vector in the conventional vector space model (VSM) [Salton 1989] by first removing its stopwords and stemming the remaining terms to their simple forms [Baeza-Yates and Ribeiro-Neto 1999]. A VSM vector is a set of tuples of terms and their weights. Although many term-weighting schemes have been proposed, no scheme has been shown to be significantly better than others for the task of event detection [Papka 1999]. In our experiments, we used the following simple TF-IDF scheme [Salton 1989], which proved effective in the TDT 2000 contest [Allan et al. 2000]:

$$
W_{t m, d}=t f_{t m, d} * \log \left(D / d f_{t m}\right),
$$

where $w_{t m, d}$ is the weight of a term $t m$ in document $d ; t f_{t m, d}$ is the term frequency (TF) of $t m$ in document $d ; \log \left(D / d f_{t m}\right)$ is the inverted document frequency (IDF) of the term $t m$; $D$ is the number of documents in the system corpus; and $d f_{t m}$ is the number of documents in the corpus in which $t m$ occurs. TF-IDF determines the weight of a term by considering both the term frequency and the inverted document frequency. Intuitively, terms with a high term frequency should have large weights. However, considering only the term frequency overestimates general terms, which results in poor distinction of documents. IDF resolves this problem by reducing the weights of general

terms. The term weights of a document are then normalized in terms of the Euclidean length [Manning et al. 2008] to represent the document as a unit vector.

ACM Transactions on Information Systems, Vol. 27, No. 2, Article 9, Publication date: February 2009. 
We adopt the noncentroid approach to represent the context of a detected event as a set of related documents. Papka [1999] and Leuski and Allan [2002] showed that, compared to centroid-based representation, the noncentroid approach generally achieves a superior event detection performance. The similarity between an incoming document $d$ and a cluster $c$ is then defined as the maximum cosine value between the vectors of the document and the constituent documents of the cluster. In addition to the set of documents, each cluster $c$ in LIPED has four variables: c.threshold, c.output, c. $\rho$, and c. $\delta$. Briefly, c.threshold $\in[0,1]$ is the clustering threshold between a document and cluster $c$; and c.output $\in K$ records the observed outcome of cluster $c$ in the current time slot. In this study, the observed outcome is the number of the clustered documents. The variables $c . \rho$ and $c . \delta$ record the probabilities of the cluster $c$ associated with life profiles and states. These probabilities, together with c.output, are used for status maintenance purposes. We discuss the usage of these variables in Section 4.2.

\subsection{Life Profile-Based Event Detection}

To deploy LIPED, an event detection method only requires the function StatusMaintenance shown in Figure 4 to update the clustering threshold periodically. Figure 3 shows the LIPED procedure using an incremental clustering algorithm. Initially, a set of life profiles $L P$ are trained by the method described in Section 3, and used to predict the activeness status of detected events. During the detection process, each incoming document $d$ is added to a cluster $c$ if the similarity between $d$ and $c$ is greater than the clustering threshold c.threshold. The algorithm in Figure 3 is a soft clustering procedure [Franz et al. 2001], whereby a document can belong to more than one cluster; however, it can be easily changed to a hard clustering procedure in which a document can only belong to the cluster with the maximum similarity. If no cluster satisfies the threshold constraint, a cluster representing a newly detected event is created so that the document $d$ is the first document. After assigning a document to a cluster, we increase the cluster's c.output by one to update the observed outcome. After all the documents generated in a time slot have been processed, each cluster executes the function StatusMaintenance shown in Figure 4 to adjust its activeness status according to the observed outcome.

The StatusMaintenance procedure is a modification of the Viterbi algorithm [Viterbi 1967], which calculates the best state transition sequence of an outcome sequence against a HMM. LIPED only requires the current state of the best transition sequence for threshold adjustment. Therefore, we use the optimal substructure [Cormen et al. 2001] of the Viterbi algorithm, that is, "the best state of the current time slot can be obtained by consulting the best state of the last time slot," to calculate the probabilities related to the current state in two $L \times N$ matrices, $c . \rho$ and $c . \delta$. The element $c . \rho[l, i]$ stores the total probability that cluster $c$ belongs to life profile $l$ and currently locates in state $i$, while the element $c . \delta[l, i]$ stores $c$ 's highest probability of state transitions that currently end in state $i$ of life profile $l$. When a cluster is created, c. $\rho$ and $c . \delta$ are initialized as $c . \rho[l, i]=c . \delta[l, i]=\pi_{i}^{l} * b_{i, \text { c.output }}^{l}$, where $1 \leq l \leq L$ and $1 \leq i \leq N$. 


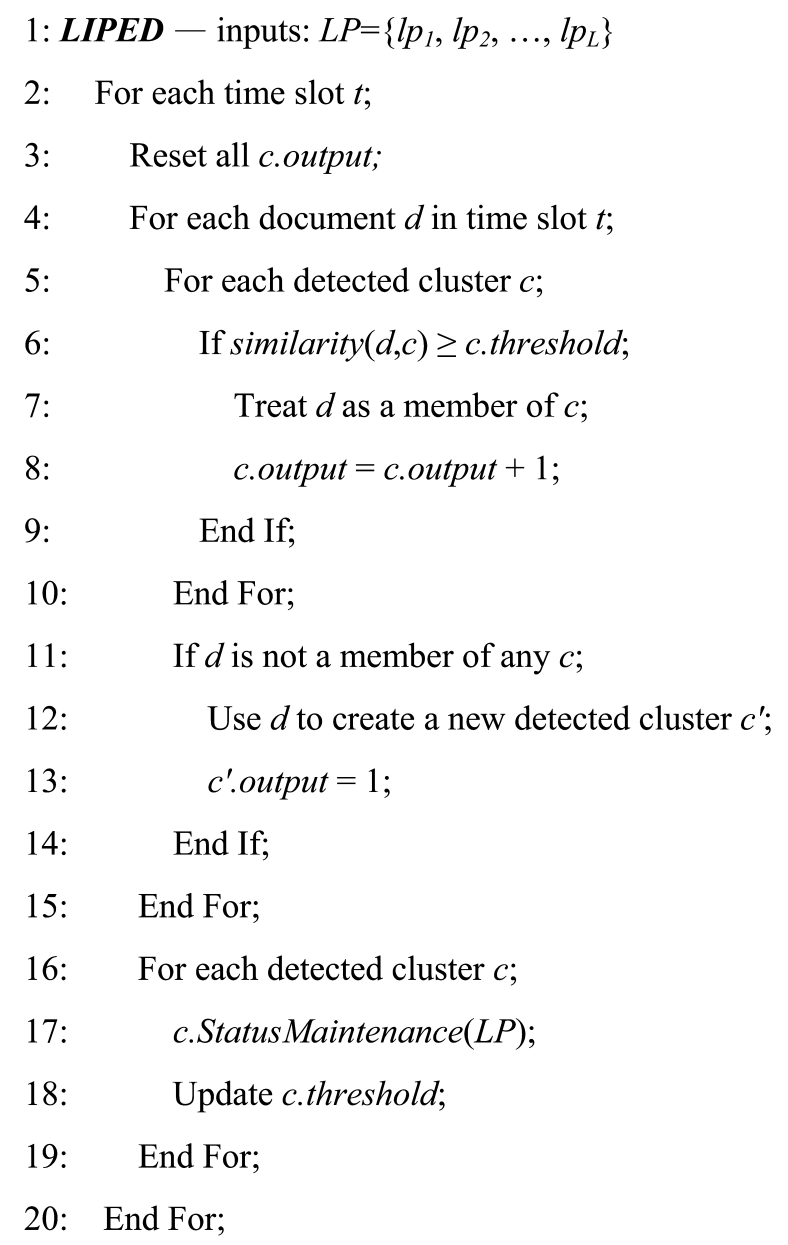

Fig. 3. LIfe Profile-based Event Detection (LIPED) using the incremental clustering algorithm.

The superscript $l$ designates the profile index that a profile component belongs to. When a cluster executes the function StatusMaintenance, the matrices are updated to reflect the activeness status of the detected event based on the observed outcome (lines 6 and 7 of Figure 4). Note that c. $\rho[l, i]$ is updated by summing up the probabilities of all possible departing states in the last time slot, multiplied by the state transition probabilities and the outcome symbol probability with respect to the observed outcome, but $c . \delta[l, i]$ only considers the highest probability. With these two matrices, the likelihood between a cluster $c$ and a life profile $l$, likelihood $d_{c}^{l}$, is the summation of $c . \rho[l, i]$ for all states, that is, likelihood $d_{c}^{l}=\Sigma_{i=1}^{N} c_{.} \rho[l, i]$; the most likely life profile of the cluster $\hat{l}_{c}$ is the one with the maximal likelihood, that is, $\hat{l}_{c}=\arg \max _{l=1}^{L}$ likelihood $_{c}^{l}$; and the cluster's activeness status for a life profile $l, \hat{S}_{c}^{l}$, is $\operatorname{argmax}_{i=1}^{N}$ c. $\delta[l, i]$. 


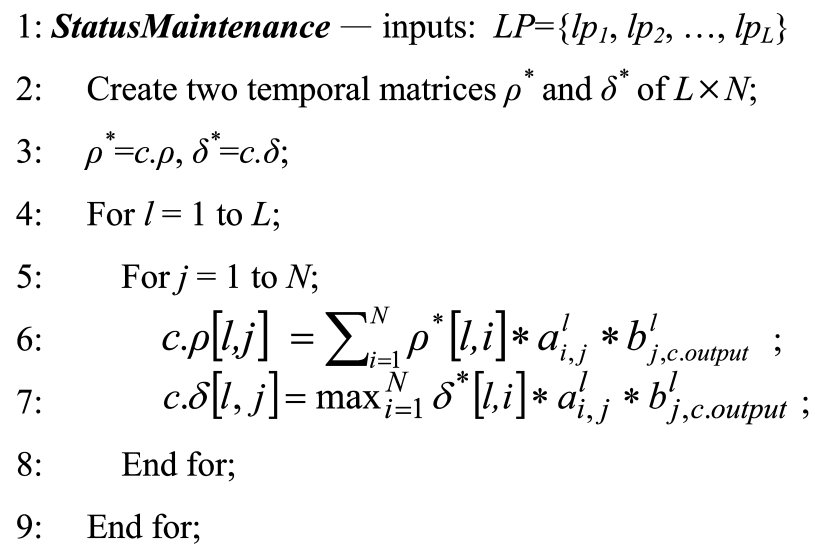

Fig. 4. Status maintenance of detected event.

The function StatusMaintenance requires $O(N L)$ space, since it allocates two $L \times N$ matrices to each cluster, and its computation cost is $O\left(N^{2} L\right)$. Even though the quadratic property makes it difficult to execute the function on the fly, StatusMaintenance is only executed once per time slot, which is practical.

\subsection{Threshold Strategies}

After the probabilities related to activeness statuses have been updated, the clustering threshold of a cluster is adjusted, as shown in line 18 of Figure 3. Each state in a life profile is assigned a clustering threshold as follows:

$$
\operatorname{thd}(i)=t h d_{\text {top }}-(i-1) *\left(t h d_{t o p}-t h d_{\text {down }}\right) / N,
$$

where $i$ is the state index, $t h d_{t o p}$ and $t h d_{\text {down }}$ are framework parameters that specify the adaptive threshold range, and $0 \leq t h d_{d o w n} \leq t h d_{t o p} \leq 1$. The formula follows the burst-and-diverse principle by assigning lower thresholds to active states. We investigate two threshold strategies in this study. The first, called the smoothed threshold, determines the threshold of a cluster by averaging the thresholds of all potential future states of all life profiles as follows:

$$
\text { c.threshold }=\sum_{i=1}^{l} \text { likelihood_ratio }{ }_{c}^{l} *\left(\sum_{j=1}^{N} a_{\hat{s}_{c, l}^{l}}^{l} * \operatorname{thd}(j)\right),
$$

where likelihood_ratio ${ }_{c}^{l}=$ likelihood $_{c}^{l} / \Sigma_{l^{\prime}=1}^{L}$ likelihood $_{c}^{l^{\prime}}$.

The second strategy, called the most-likely threshold, determines the threshold of a cluster by considering the most likely future state of the most likely profile as follows:

$$
\text { c.threshold }=\text { thd }\left(\arg \max _{j=1}^{N} a_{\hat{s}_{c, j}^{c_{c}}}^{l_{c}}\right) .
$$

Compared to the most-likely threshold strategy, the smoothed threshold strategy is a relatively conservative approach in which every life profile has 
Table I. The Contingency Table for Metric Definitions

\begin{tabular}{|l|c|c|}
\hline & In Labeled Event & Not in Labeled Event \\
\hline In cluster & $a$ & $b$ \\
\hline Not in cluster & $c$ & $d$ \\
\hline
\end{tabular}

an effect on determining the clustering threshold. In our experiments, we investigate the properties of these two strategies.

\section{EMPIRICAL EVALUATIONS}

In this section, we first introduce the data corpus, performance metrics, and evaluation process. Then, the LIPED framework is applied to several popular event detection algorithms to demonstrate the advantages of life profile modeling. Finally, the time complexity of LIPED is analyzed and examples of detecting burst-and-diverse events are given.

\subsection{Data Corpus and Performance Metrics}

The Linguistic Data Consortium ${ }^{2}$ has compiled a series of TDT corpora for the annual TDT contests. Conventionally, the training data for a contest must be the labeled events of previous contests used to determine the robustness of evaluated methods. In a previous work [Chen et al. 2005], we found from experiments that a small number of training events would cause the learned life profiles to be overfitted. Therefore, in this paper, we aggregate the labeled events in the TDT1, TDT2, and TDT3 corpora for the construction and validation of HMM-based life profiles, and evaluate the performance of LIPED on the official TDT4 corpus. The TDT4 corpus comprises the sets of English, Arabic, and Chinese news documents from several well-known news agencies for the period October 1, 2000, to January 31, 2001. We only used the set of English texts for evaluation, which consists of 28,390 news documents from eight popular news agencies. Among them, seventy news events with 1,926 related documents were labeled for various evaluation tasks. The evaluations follow the TDT contest rules for comparing the performance of different methods. First, each method detects events (also called clusters) and their related documents from the corpus. Note that each document can belong to only one cluster. Next, each of the seventy labeled events is compared with the resulting clusters, and the best matched cluster is selected for microaverage evaluation. Note that the degree of match between a labeled event and a cluster is determined by the number of overlapping documents.

We evaluate the performance of event detection based on the following six metrics: precision $(p)$, recall $(r)$, miss $(m s)$, false alarm $(f)$, F1-measure $(F 1)$, and normalized cost $\left(\operatorname{cost}_{n}\right)$. In terms of the above contingency table, precision $p=a /(a+b)$ is the fraction of clustered documents that are related; and recall $r=a /(a+c)$ is the fraction of related documents that are clustered. Precision and recall are two important measurements for evaluating the effectiveness of an information retrieval system. A high degree of precision indicates that the

\footnotetext{
${ }^{2} \mathrm{http} / / / w w w . l d c . u p e n n . e d u /$ 
system can generate cohesive document clusters, while high recall means that the detected clusters cover a high percentage of documents of the labeled events. Generally, precision and recall are inversely related, that is, when the precision increases, recall typically declines, and vice versa [Frakes and Baeza-Yates 1992]. Hence, precision and recall by themselves are not good indicators of the effectiveness of global performance. On the other hand, the F1-measure, $F 1=$ $2 p r /(p+r)$, is considered an objective measure because it balances precision and recall. Tasks in TDT are evaluated based on error rates, such as misses and false alarms [Fiscus and Doddington 2002; Manmatha et al. 2002]. Miss $m s=c /(a+c)$ is the fraction of related documents that are not clustered, and is the counterpart of recall. False alarm $f=b /(b+d)$ is the fraction of unrelated documents that are clustered, and is the ratio of false positives. Similar to the precision and recall, the miss and false alarm metrics vary inversely; and their linear combination, the normalized cost shown in Equation (11), is regarded as the official measure in TDT [Manmatha et al. 2002].

$$
\begin{aligned}
\operatorname{cost}_{n}= & C_{\text {Miss }} * m s * P_{\text {target }}+C_{F A} * f *\left(1-P_{\text {target }}\right) / \\
& \min \left\{C_{\text {Miss }} * P_{\text {target }}, C_{F A} *\left(1-P_{\text {target }}\right)\right\}
\end{aligned}
$$

In Equation (11), the values of $C_{M i s s}, C_{F A}$, and $P_{\text {target }}$ are 1, 0.1, and 0.02, respectively. Systems with low costs are obviously superior, since they are less likely to make detection mistakes. These error rates, especially the normalized cost, have received more attention from the TDT community than the precision, recall, and F1 metrics because TDT scholars do not consider event detection as a traditional information retrieval task; therefore, the normalized cost value is used to determine the superiority of compared methods [Manmatha et al. 2002].

To demonstrate the advantages of incorporating LIPED into event detection algorithms, we evaluate the framework on three well-known event detection algorithms, namely the time window method (WND) [Yang et al. 1998], the time-based threshold method (TBT) [Allan et al. 1998a], and the incremental clustering algorithm (INC) [Rijsbergen 1979]. While these methods are simple, they have proven very effective in TDT contests, especially the time window method. Zhang et al. [2004] noted that the time window method with the simple TF-IDF term weighting scheme and cosine similarity metric was the top performing system in the TDT 2002 and 2003 official evaluations. Their experiments show that the time window method can achieve excellent detection performances compared with their method. More recently, Chou and Chen [2008] observed that the time window method is highly comparable to the proposed probabilistic method. The three event detection algorithms are implemented as described in the respective papers without incorporating sophisticated refinements, such as using background knowledge for proper noun extraction [Hatzivassiloglou et al. 2000; Makkonen et al. 2004] and the parameter training process [Chen et al. 2003]. As a result, the evaluation process can be replicated, and we can focus on the effects of the proposed life profile modeling technique. In the following subsections, we use the suffix "-L" to indicate that a method incorporates LIPED. For instance, the notation WND-L stands for the time window method incorporating LIPED. 
The evaluation process involves the following steps. First, each of the three methods performs a series of event detection tasks with the clustering threshold set between 0.0 and 1.0, and increased in increments of 0.01 for each task. (However, the experiment results show that the meaningful threshold range is only from 0.2 to 0.4.) For each threshold setting, we also incorporate LIPED with the original method to compare the performance with and without life profile modeling. The value of the framework parameter $t h d_{t o p}$ is identical to the threshold of the original method and $t h d_{d o w n}$ is acquired from a validation process, which we described in the next section. In Section 5.3, the best result of each original method is taken as the baseline to examine the effects of LIPED from various perspectives. As an optimal threshold may not be obtainable, we present the complete comparisons for a range of threshold settings in Section 5.3.4. The results show that methods incorporating LIPED always outperform the baseline methods and the improvements are statistically significant in terms of the $T$-test examination.

\subsection{Life Profile Preparation}

We partition the labeled events of TDT1, TDT2, and TDT3 into two parts for training and validation [Mitchell 1997]. Excluding the events that last only one day, which are too short to train HMMs, there are 276 events. We randomly select 30 events from the TDT3 corpus for validation and the remaining 246 events are used for training. Each training event is represented as a sequence of the number of related documents published per day. The hierarchical agglomerative clustering (HAC) algorithm is then applied to the resulting sequences to form a class hierarchy. For HAC, we consider three well-known inter-cluster similarity strategies, namely, single-link, complete-link, and average-link strategies [Salton 1989]. In addition, to obtain the similarity (i.e., distance) between two sequences, we apply the dynamic time warping (DTW) algorithm [Myers et al. 1980], which is widely used for measuring the distance between two time series of different length. As a result, a node in the hierarchy can be regarded as a life profile of the constituent sequences. The deeper the node is in the hierarchy, the more specific the profile will be. In the experiments, LIPED runs on each of the top four levels, from the root (i.e., level 0) down to the level 3, of the profile hierarchy to assess the influence of the profile's granularity on event detection. The performance of level 0 can be considered the same as that of Aizen's method [Aizen et al. 2004] because, like Aizen's method, it combines all activeness trends in the training data into a single life profile. In our evaluations, the number of states of a life profile is set at 20 .

The validation process is identical to the evaluation process except that for each $t h d_{\text {top }}, t h d_{\text {down }}$ is set as $\left(t h d_{t o p}-X\right)$, where $X=0.03,0.05,0.1$, and 0.15 respectively; and the $t h d_{d o w n}$ value that achieves the best cost and F1 scores is selected as the threshold range used in the evaluation process.

\subsection{Performance Evaluations}

5.3.1 LIPED on the Time Window Method. For the time window method, the window size is 3000 documents and the decaying similarity [Yang et al. 
Table II. The Results of the Time Window Methods with LIPED

\begin{tabular}{|c|c|c|c|c|c|c|}
\hline & $p$ & $r$ & $F 1$ & $m s$ & $f(\%)$ & cost $_{n}$ \\
\hline WND-0.36 & 0.6879 & 0.5030 & 0.5811 & 0.4970 & 0.0212 & 0.4980 \\
\hline WND-0.34 & 0.5593 & 0.5447 & 0.5519 & 0.4553 & 0.0399 & 0.4572 \\
\hline S-L, L0, ST(Aizen) & 0.6392 & 0.5165 & 0.5713 & 0.4835 & 0.0271 & $0.4848^{* * *}$ \\
\hline S-L, L1, ST & 0.6393 & 0.5360 & 0.5831 & 0.4640 & 0.0281 & $0.4653^{* * *}$ \\
\hline S-L, L2, ST & 0.6378 & 0.5393 & $0.5844^{*}$ & 0.4607 & 0.0285 & $0.4621^{* * *}$ \\
\hline S-L, L3, ST & 0.6389 & 0.5398 & $0.5852^{*}$ & 0.4602 & 0.0284 & $0.4615^{* * *}$ \\
\hline C-L, L0, ST(Aizen) & 0.6392 & 0.5165 & 0.5713 & 0.4835 & 0.0271 & $0.4848^{* * *}$ \\
\hline C-L, L1, ST & 0.6440 & 0.5382 & $0.5864^{*}$ & 0.4618 & 0.0277 & $0.4631^{* * *}$ \\
\hline C-L, L2, ST & 0.6446 & 0.5339 & $0.5841^{*}$ & 0.4661 & 0.0273 & $0.4674^{* * *}$ \\
\hline C-L, L3, ST & 0.6428 & 0.5344 & $0.5836^{*}$ & 0.4656 & 0.0276 & $0.4669^{* * *}$ \\
\hline A-L, L0, ST(Aizen) & 0.6392 & 0.5165 & 0.5713 & 0.4835 & 0.0271 & $0.4848^{* * *}$ \\
\hline A-L, L1, ST & 0.6413 & 0.5339 & 0.5827 & 0.4661 & 0.0278 & $0.4675^{* * *}$ \\
\hline A-L, L2, ST & 0.6391 & 0.5404 & $0.5856^{*}$ & 0.4596 & 0.0284 & $0.4610^{* * *}$ \\
\hline A-L, L3, ST & 0.6401 & 0.5350 & 0.5828 & 0.4650 & 0.0280 & $0.4664^{* * *}$ \\
\hline S-L, L0, MT(Aizen) & 0.6359 & 0.5187 & 0.5713 & 0.4813 & 0.0276 & $0.4826^{* * *}$ \\
\hline S-L, L1, MT & 0.6359 & 0.5236 & 0.5743 & 0.4764 & 0.0279 & $0.4778^{* *}$ \\
\hline S-L, L2, MT & 0.6430 & 0.5388 & $0.5863^{*}$ & 0.4612 & 0.0278 & $0.4626^{* * *}$ \\
\hline S-L, L3, MT & 0.5847 & 0.5388 & 0.5608 & 0.4612 & 0.0356 & $0.4630^{* * *}$ \\
\hline C-L, L0, MT(Aizen) & 0.6359 & 0.5187 & 0.5713 & 0.4813 & 0.0276 & $0.4826^{* * *}$ \\
\hline C-L, L1, MT & 0.6353 & 0.5241 & 0.5744 & 0.4759 & 0.0280 & $0.4772^{* *}$ \\
\hline C-L, L2, MT & 0.6397 & 0.5409 & $0.5862^{*}$ & 0.4591 & 0.0283 & $0.4604^{* * *}$ \\
\hline C-L, L3, MT & 0.6389 & 0.5409 & $0.5859^{*}$ & 0.4591 & 0.0284 & $0.4604^{* * *}$ \\
\hline A-L, L0, MT(Aizen) & 0.6359 & 0.5187 & 0.5713 & 0.4813 & 0.0276 & $0.4826^{* * *}$ \\
\hline A-L, L1, MT & 0.6403 & 0.5404 & $0.5861^{*}$ & 0.4596 & 0.0282 & $0.4610^{* * *}$ \\
\hline A-L, L2, MT & 0.6386 & 0.5393 & $0.5848^{*}$ & 0.4607 & 0.0284 & $0.4621^{* * *}$ \\
\hline A-L, L3, MT & 0.6389 & 0.5398 & $0.5852^{*}$ & 0.4602 & 0.0284 & $0.4615^{* * *}$ \\
\hline
\end{tabular}

Legend: WND-\#: the original time window method with \# similarity threshold. S/C/A-L: single/complete/averagelink. L\#: level \# of profiles in the profile hierarchy. ST: the smoothed threshold strategy. MT: the most-likely threshold strategy. $t h d_{t o p}=0.36$. $t h d_{d o w n}=t h d_{t o p}-0.10$. The results marked with *, **, and *** show, respectively, improvements over the baseline with $90 \%, 95 \%$, and $99 \%$ confidence levels based on the one-tailed paired T-test.

1998 is employed to give temporally close documents more influence than those farther apart. Our experiment results show that the time window method achieves the best performance for the normalized cost metric (0.2671) when the clustering threshold is set at 0.20 . However, the corresponding precision is 0.26 , which implies that three out of four of the detected documents are irrelevant. Obviously, the detected events are too noisy to be considered as good detection results. We found from the experiments that the false alarm rate is much lower than the miss rate. Even though the cost function augments the penalty of false alarms nearly five times to misses, the miss rate still dominates the cost metric in the experiments. For the sake of low cost, a compared method can simply relax the clustering threshold to obtain a low miss rate in spite of the unacceptable precision. To ensure that the comparison is reasonable and fair, in the following sub-sections, we take the best $\mathrm{F} 1$ performance of each original method as the baseline and investigate the improvements when LIPED with various life profiles and threshold strategies is incorporated.

Table II shows the results of the time window methods that incorporate LIPED for various settings. The best results of each metric are highlighted in 


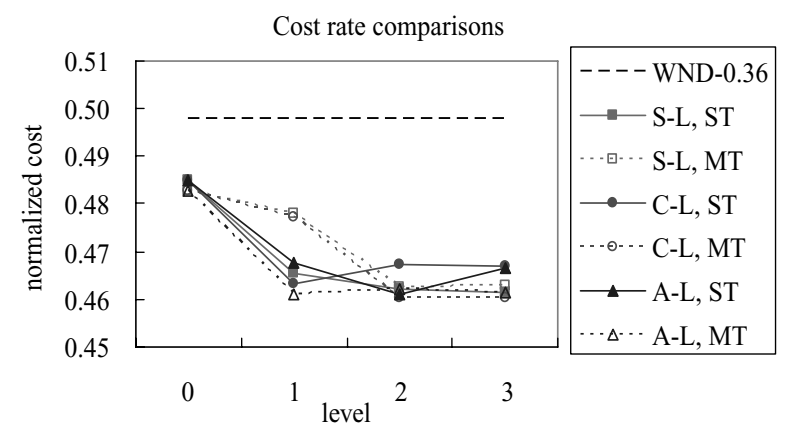

Fig. 5. Cost vs. profile level of the time window method.

gray shadow. In our experiment, the best F1 score of the time window method occurs when the threshold is set at 0.36 and used as the baseline for comparison. The declining precision and the rising false alarm rates of the methods that incorporate LIPED indicate that the relaxed threshold for active events may lead to misclustering of noisy documents; however, the improvement in the miss rate is significant, that is, approximately $7.6 \%$ (from 0.497 to 0.459 ) for \{C-L, L2, MT\}. Moreover, to achieve a miss score of 0.459 , the threshold of the original method has to be relaxed to 0.34 (i.e., WND-0.34), which results in a low precision rate of 0.559 and a high false alarm rate of 0.000399 . In contrast, the methods with LIPED achieve better scores of 0.639 and 0.000283 , which represent $15.3 \%$ and $32.1 \%$ improvements, respectively. Briefly, for nearly all experiment settings, methods with LIPED perform better in terms of the normalized cost (7.5\% improvement) and F1 scores than the baseline method, even when a single life profile (i.e., Aizen's method) is used. Furthermore, the improvements are statistically significant in terms of the one-tailed paired $T$-test examination.

Figure 5 shows that the profiles with different intercluster similarity strategies (i.e., single/complete/average-link) achieve similar detection performances in terms of the normalized cost score. In addition, the most likely threshold strategy performs better than the smoothed threshold strategy, since it usually generates a lower threshold than the smoothed threshold strategy, and thus yields better miss and normalized cost scores, as shown in Table II. The figure also indicates that, in terms of the normalized cost, methods with more specific profiles (e.g., at levels 1, 2, and 3 in this experiment) perform better than those of level 0 , and methods at L2 generally achieve the best performance. The reason is that the life profiles of level 0 are too general and do not consider the distinct activeness trends of constituent events, which degrades the detection performance.

5.3.2 LIPED on the Time-Based Threshold Method. As shown in Table III, the baseline of the time-based threshold method occurs when the threshold is set at 0.34 with a 0.0003 time penalty [Allan et al. 1998a]. Similar to the results of the time window method, TBT-L sacrifices minor declines in precision and false alarm rates in return for substantial improvements in the miss rate 
Table III. Results of the Time-Based Threshold Methods with LIPED

\begin{tabular}{|c|c|c|l|l|l|c|}
\hline & $p$ & $r$ & $F 1$ & $m s$ & $f(\%)$ & cost $_{n}$ \\
\hline TBT-0.34 & 0.7020 & 0.4840 & 0.5730 & 0.5160 & 0.0191 & 0.5169 \\
\hline TBT-0.32 & 0.6207 & 0.5144 & 0.5625 & 0.4856 & 0.0292 & 0.4870 \\
\hline S-L, L0, ST(Aizen) & 0.6890 & 0.5019 & $0.5807^{* *}$ & 0.4981 & 0.0211 & $0.4991^{* * *}$ \\
\hline S-L, L1, ST & 0.6843 & 0.5051 & $0.5812^{* * *}$ & 0.4949 & 0.0217 & $0.4959^{* * *}$ \\
\hline S-L, L2, ST & 0.6800 & 0.5068 & $0.5807^{* *}$ & 0.4932 & 0.0222 & $0.4943^{* * *}$ \\
\hline S-L, L3, ST & 0.6932 & 0.5057 & $0.5848^{* * *}$ & 0.4943 & 0.0208 & $0.4953^{* * *}$ \\
\hline C-L, L0, ST(Aizen) & 0.6890 & 0.5019 & $0.5807^{* *}$ & 0.4981 & 0.0211 & $0.4991^{* * *}$ \\
\hline C-L, L1, ST & 0.6795 & 0.5079 & $0.5813^{* * *}$ & 0.4921 & 0.0223 & $0.4932^{* * *}$ \\
\hline C-L, L2, ST & 0.6792 & 0.5084 & $0.5815^{* * *}$ & 0.4916 & 0.0223 & $0.497^{* * *}$ \\
\hline C-L, L3, ST & 0.6777 & 0.5084 & $0.5810^{* * *}$ & 0.4916 & 0.0225 & $0.4927^{* * *}$ \\
\hline A-L, L0, ST(Aizen) & 0.6890 & 0.5019 & $0.5807^{* *}$ & 0.4981 & 0.0211 & $0.4991^{* * *}$ \\
\hline A-L, L1, ST & 0.6859 & 0.5089 & $0.5843^{* * *}$ & 0.4911 & 0.0217 & $0.4921^{* * *}$ \\
\hline A-L, L2, ST & 0.6800 & 0.5079 & $0.5814^{* * *}$ & 0.4921 & 0.0222 & $0.4932^{* * *}$ \\
\hline A-L, L3, ST & 0.6792 & 0.5084 & $0.5815^{* * *}$ & 0.4916 & 0.0223 & $0.4927^{* * *}$ \\
\hline S-L, L0, MT(Aizen) & 0.6819 & 0.5019 & $0.5782^{* *}$ & 0.4981 & 0.0218 & $0.4991^{* * *}$ \\
\hline S-L, L1, MT & 0.6811 & 0.5117 & 0.5843 & 0.4883 & 0.0223 & $0.4894^{* * *}$ \\
\hline S-L, L2, MT & 0.6821 & 0.5106 & $0.5840^{* *}$ & 0.4894 & 0.0221 & $0.4905^{* * *}$ \\
\hline S-L, L3, MT & 0.6850 & 0.5057 & $0.5819^{* *}$ & 0.4943 & 0.0216 & $0.4953^{* * *}$ \\
\hline C-L, L0, MT(Aizen) & 0.6819 & 0.5019 & $0.5782^{* *}$ & 0.4981 & 0.0218 & $0.4991^{* * *}$ \\
\hline C-L, L1, MT & 0.6804 & 0.5111 & $0.5837^{* *}$ & 0.4889 & 0.0223 & $0.4900^{* * *}$ \\
\hline C-L, L2, MT & 0.6791 & 0.5117 & $0.5836^{* *}$ & 0.4883 & 0.0225 & $0.4894^{* * *}$ \\
\hline C-L, L3, MT & 0.6791 & 0.5117 & $0.5836^{* *}$ & 0.4883 & 0.0225 & $0.4894^{* * *}$ \\
\hline A-L, L0, MT(Aizen) & 0.6819 & 0.5019 & $0.5782^{* *}$ & 0.4981 & 0.0218 & $0.4991^{* * *}$ \\
\hline A-L, L1, MT & 0.6840 & 0.5068 & $0.5822^{* * *}$ & 0.4932 & 0.0218 & $0.4943^{* * *}$ \\
\hline A-L, L2, MT & 0.6806 & 0.5117 & $0.5842^{* *}$ & 0.4883 & 0.0223 & $0.4894^{* * *}$ \\
\hline A-L, L3, MT & 0.6842 & 0.5084 & 0.5833 & 0.4916 & 0.0218 & $0.4926^{* * *}$ \\
\hline
\end{tabular}

Legend: TBT-\#: the original time-based threshold method with \# similarity threshold. $t h d_{\text {top }}=0.34$. thd $d_{\text {down }}=t h d_{\text {top }}-0.15$.

(from 0.516 to 0.488 or $5.3 \%$ ) and the recall rate (from 0.484 to 0.511 or $5.7 \%$ ); hence, the normalized cost and F1 are also improved. In addition, to achieve a similar miss rate to that of LIPED, the original method has to relax the clustering threshold to 0.32 . However, this low threshold leads to an inferior false alarm rate $(0.000292)$ and precision rate $(0.620)$, while TBT-L achieves better scores of 0.000223 and 0.681 , which represent improvements of $28.8 \%$ and $11.7 \%$, respectively. Even though the smoothed threshold strategy yields the best F1 score, the most likely threshold strategy generally achieves superior cost performances. In sum, the improvement in detection is about $5.3 \%$ for the normalized cost score when LIPED is incorporated into the time-based threshold method. Similar to the last experiment, Figure 6 shows that methods with LIPED perform generally better than the baseline method.

5.3.3 LIPED on the Incremental Clustering Algorithm. The incremental clustering algorithm generates a balanced detection result when the threshold is set at 0.40. Table IV shows the improvements when LIPED is incorporated. Again, INC-L achieves 5.9\% and 4.1\% improvements in the miss and recall rates, respectively, and similar false alarm and precision rates to the baseline method. Consequently, INC-L yields better F1 scores and achieves a 5.7\% improvement in the normalized cost score. Comparing the results with similar 


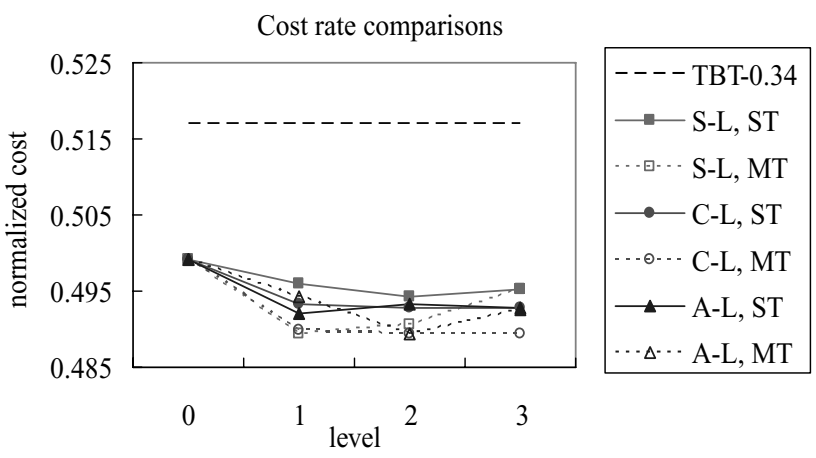

Fig. 6. Cost vs. profile level of the time-based threshold method.

Table IV. The Results of the Incremental Clustering Algorithms with LIPED

\begin{tabular}{|c|l|l|l|l|l|l|}
\hline & \multicolumn{1}{|c|}{$p$} & \multicolumn{1}{|c|}{$r$} & \multicolumn{1}{|c|}{$F 1$} & \multicolumn{1}{c|}{$f(\%)$} & cost $_{n}$ \\
\hline INC-0.40 & 0.2654 & 0.5892 & 0.3659 & 0.4108 & 0.1516 & 0.4182 \\
\hline INC-0.39 & 0.1898 & 0.6233 & 0.2910 & 0.3767 & 0.2473 & 0.3888 \\
\hline S-L, L0, ST(Aizen) & 0.2642 & 0.5984 & $0.3665^{* * *}$ & 0.4016 & 0.1549 & $0.4092^{* * *}$ \\
\hline S-L, L1, ST & 0.2664 & 0.6065 & 0.3702 & 0.3935 & 0.1552 & $0.4011^{* *}$ \\
\hline S-L, L2, ST & 0.2676 & 0.6098 & $0.3720^{*}$ & 0.3902 & 0.1551 & $0.3978^{* *}$ \\
\hline S-L, L3, ST & 0.2670 & 0.6081 & 0.3710 & 0.3919 & 0.1552 & $0.3994^{* *}$ \\
\hline C-L, L0, ST(Aizen) & 0.2642 & 0.5984 & $0.3665^{* * *}$ & 0.4016 & 0.1549 & $0.4092^{* * *}$ \\
\hline C-L, L1, ST & 0.2668 & 0.6081 & 0.3708 & 0.3919 & 0.1553 & $0.3995^{* *}$ \\
\hline C-L, L2, ST & 0.2678 & 0.6092 & $0.3721^{*}$ & 0.3908 & 0.1548 & $0.3983^{* *}$ \\
\hline C-L, L3, ST & 0.2673 & 0.6098 & $0.3717^{*}$ & 0.3902 & 0.1553 & $0.3978^{* *}$ \\
\hline A-L, L0, ST(Aizen) & 0.2642 & 0.5984 & $0.3665^{* * *}$ & 0.4016 & 0.1549 & $0.4092^{* * *}$ \\
\hline A-L, L1, ST & 0.2667 & 0.6070 & 0.3706 & 0.3930 & 0.1551 & $0.4005^{* *}$ \\
\hline A-L, L2, ST & 0.2677 & 0.6098 & $0.3721^{*}$ & 0.3902 & 0.1550 & $0.3978^{* *}$ \\
\hline A-L, L3, ST & 0.2675 & 0.6098 & $0.3718^{*}$ & 0.3902 & 0.1552 & $0.3978^{* *}$ \\
\hline S-L, L0, MT(Aizen) & 0.2648 & 0.5989 & $0.3672^{* *}$ & 0.4011 & 0.1545 & $0.4086^{* * *}$ \\
\hline S-L, L1, MT & 0.2672 & 0.6098 & $0.3715^{*}$ & 0.3902 & 0.1554 & $0.3978^{* *}$ \\
\hline S-L, L2, MT & 0.2661 & 0.6119 & $0.3709^{*}$ & 0.3881 & 0.1568 & $0.3957^{* *}$ \\
\hline S-L, L3, MT & 0.2669 & 0.6070 & 0.3708 & 0.3930 & 0.1549 & $0.4005^{* *}$ \\
\hline C-L, L0, MT(Aizen) & 0.2648 & 0.5989 & $0.3672^{* *}$ & 0.4011 & 0.1545 & $0.4086^{* * *}$ \\
\hline C-L, L1, MT & 0.2673 & 0.6098 & $0.3717^{*}$ & 0.3902 & 0.1553 & $0.3978^{* *}$ \\
\hline C-L, L2, MT & 0.2665 & 0.6136 & $0.3716^{*}$ & 0.3865 & 0.1569 & $0.3941^{* *}$ \\
\hline C-L, L3, MT & 0.2672 & 0.6098 & $0.3715^{*}$ & 0.3902 & 0.1554 & $0.3978^{* *}$ \\
\hline A-L, L0, MT(Aizen) & 0.2648 & 0.5989 & $0.3672^{* *}$ & 0.4011 & 0.1545 & $0.4086^{* * *}$ \\
\hline A-L, L1, MT & 0.2668 & 0.6081 & 0.3708 & 0.3919 & 0.1553 & $0.3995^{* *}$ \\
\hline A-L, L2, MT & 0.2677 & 0.6098 & $0.3721^{*}$ & 0.3902 & 0.1550 & $0.3978^{* *}$ \\
\hline A-L, L3, MT & 0.2672 & 0.6098 & $0.3716^{*}$ & 0.3902 & 0.1554 & $0.3978^{* *}$ \\
\hline
\end{tabular}

Legend: the original incremental clustering algorithm with \# similarity threshold. $t h d_{t o p}=0.4$. thd $d_{\text {down }}=t h d_{\text {top }}-0.05$.

miss rate of the original method, the adaptive threshold of INC-L achieves better detection precision ( $41.1 \%$ improvement) and a better false alarm rate (37.5\% reduction).

Figure 7 also shows significant LIPED improvements for the normalized cost metric. The life profiles of level 0 are too general and can not adequately describe the activeness trends of events, which degrades the detection performance. 


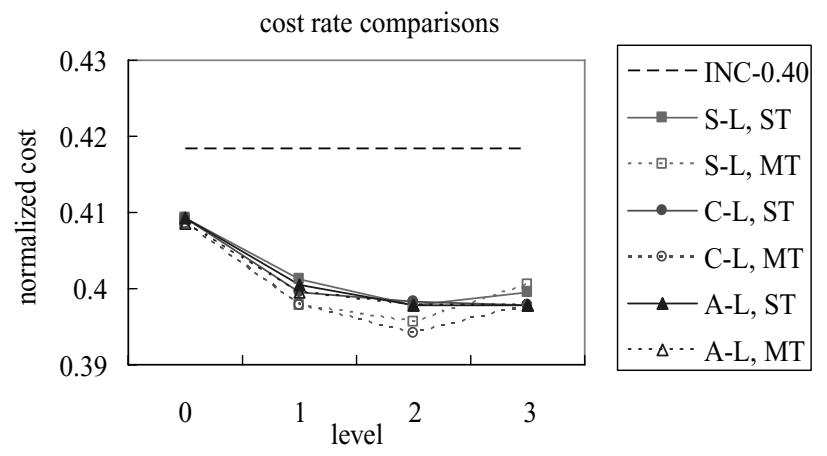

Fig. 7. Cost vs. profile level of the incremental clustering algorithm.

Table V. The Effect of the Threshold Range

\begin{tabular}{|c|c|c|c|c|c|c|}
\hline & $p$ & $r$ & $F 1$ & $m s$ & $f(\%)$ & cost $_{n}$ \\
\hline WND-0.36 & 0.6879 & 0.5030 & 0.5811 & 0.4970 & 0.0212 & 0.4980 \\
\hline WND-0.20 & 0.2768 & 0.7398 & 0.4028 & 0.2602 & 0.1797 & 0.2689 \\
\hline WND-L, range $=0.1$ & 0.6397 & 0.5409 & 0.5862 & 0.4591 & 0.0283 & 0.4604 \\
\hline WND-L, range $=0.3$ & 0.3289 & 0.7436 & 0.4561 & 0.2564 & 0.1410 & 0.2633 \\
\hline TBT-0.34 & 0.7020 & 0.4840 & 0.5730 & 0.5160 & 0.0191 & 0.5169 \\
\hline TBT-0.21 & 0.3269 & 0.6612 & 0.4375 & 0.3388 & 0.1265 & 0.3449 \\
\hline TBT-L, range $=0.15$ & 0.6791 & 0.5117 & 0.5836 & 0.4883 & 0.0225 & 0.4894 \\
\hline TBT-L, range $=0.3$ & 0.3682 & 0.6634 & 0.4736 & 0.3366 & 0.1058 & 0.3417 \\
\hline INC-0.40 & 0.2647 & 0.5908 & 0.3656 & 0.4092 & 0.1525 & 0.4167 \\
\hline INC-0.27 & 0.1395 & 0.8287 & 0.2388 & 0.1713 & 0.4750 & 0.1945 \\
\hline INC-L, range $=0.05$ & 0.2665 & 0.6136 & 0.3716 & 0.3865 & 0.1569 & 0.3941 \\
\hline INC-L, range $=0.2$ & 0.1463 & 0.8271 & 0.2486 & 0.1729 & 0.4486 & 0.1949 \\
\hline
\end{tabular}

Legend: for WND-L, $t h d_{t o p}=0.36$. For TBT-L, $t h d_{t o p}=0.34$. For INC-L, $t h d_{t o p}=0.40$. range $=\#$ : the LIPED incorporated method, where $t h d_{d o w n}=t h d_{t o p}$-\#.

5.3.4 Effects of Threshold Settings. In this subsection, we first discuss the effects of threshold ranges on LIPED and then compare the performance of the methods with different thresholds. For ease of illustration, only the results of the original methods and LIPED based on $\{\mathrm{C}-\mathrm{L}, \mathrm{L} 2, \mathrm{MT}\}$ are shown. During the validation process, we observed that while fixing the upper bound thd $d_{t o p}$, a lower $t h d_{d o w n}$, which implies a wider threshold range, results in a smaller normalized cost score. With a lower $t h d_{\text {down }}$, each state in the HMM-based life profile has a lower threshold, which helps in detecting diverse documents. Thus, both the miss rate and the normalized cost are reduced. For example, as shown in Table V, when the threshold range of WND-L is extended from 0.1 to 0.3 , the normalized cost is reduced from 0.4604 to 0.2633 , which is an improvement on the original time window method from $7.5 \%$ to $47.1 \%$. However, such a low cost does not mean the F1 score would be good because the low threshold also reduces the detection precision, and the resulting clusters would be too noisy to be considered good detection results. Nevertheless, compared to the original method with a comparable miss rate, that is, WND-0.20, WND-L with range = 0.3 still achieves better false alarm and precision improvements of $21.5 \%$ and $18.9 \%$, respectively. As a result, the F1 score improves by $13.2 \%$. 


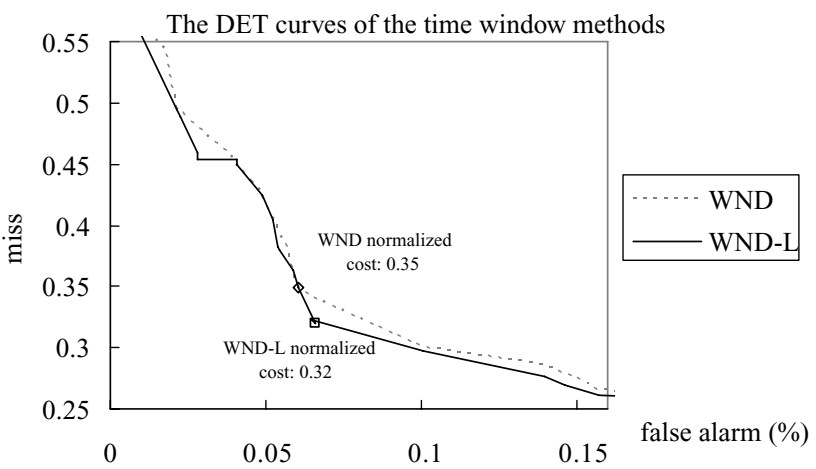

Fig. 8. The DET curves of the time window methods.

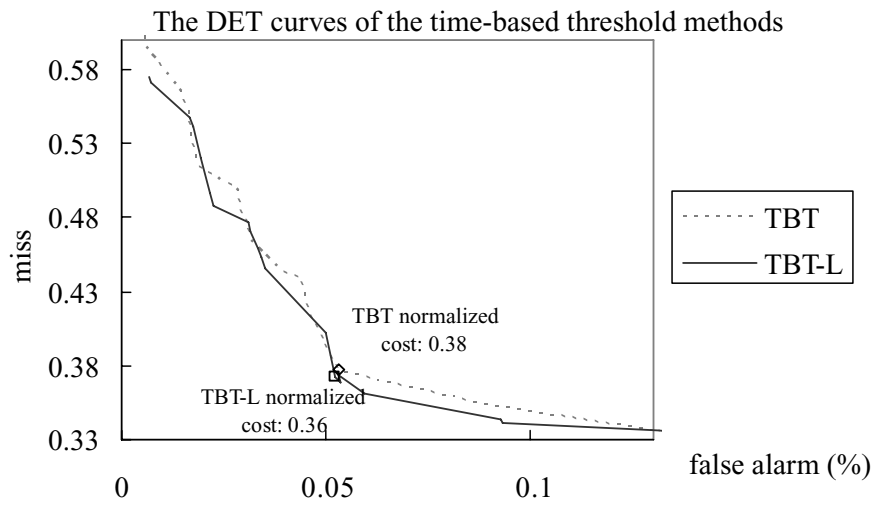

Fig. 9. The DET curves of the time-based threshold methods.

The validation process is expected to obtain a proper threshold range so that both the F1 and normalized cost of the methods with LIPED are better than those of the original methods. Generally, such a desirable situation occurs with a small threshold range for low $t h d_{t o p}$, and the range is extended moderately as $t h d_{t o p}$ increases. The threshold ranges derived from the validation process are as follows. 1) WND-L: $t h d_{d o w n}$ is set at $t h d_{t o p}-0.1$ when $t h d_{t o p}$ is in the range $[0.4,0.26]$; otherwise, $\left.t h d_{d o w n}=t h d_{\text {top }}-0.05 .2\right)$ TBT-L: $t h d_{\text {down }}$ is set at $t h d_{t o p}-0.15$ when $t h d_{t o p}$ is in the range [0.4,0.26]; otherwise, $t h d_{d o w n}=$ thd $\left.d_{\text {top }}-0.05 .3\right)$ INC-L: $t h d_{d o w n}$ is set at $t h d_{t o p}-0.05$ when $t h d_{t o p}$ is in [0.4, $0.28]$; otherwise, $t h d_{\text {down }}=t h d_{\text {top }}-0.03$.

Figures 8 to 15 show the comparisons under different threshold settings. Because miss is the counterpart of recall, we only show the miss figure. The one-tailed paired $T$-test on F1 and the normalized cost under the thresholds are given in Table VI. The $p$-values of the results indicate that the improvements made by life profile modeling are highly significant, since the methods incorporating LIPED always achieves a superior detection performance no matter what the detection threshold is given.

As shown in Figures 8 to 10, the methods with LIPED generally balance the trade-off between misses and false alarms such that their DET curves [Martin 


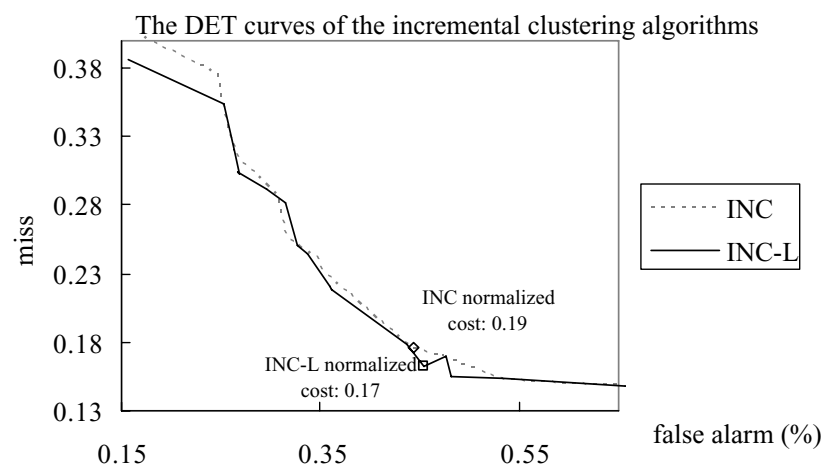

Fig. 10. The DET curves of the incremental clustering algorithms.

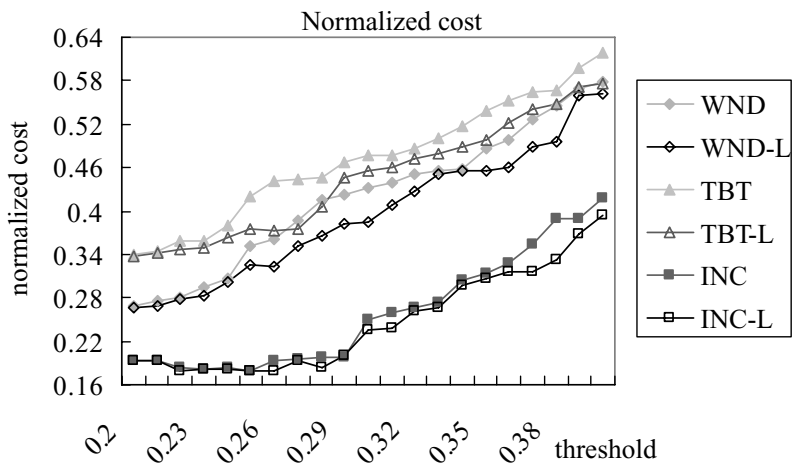

Fig. 11. The effects of thresholds on the normalized cost.

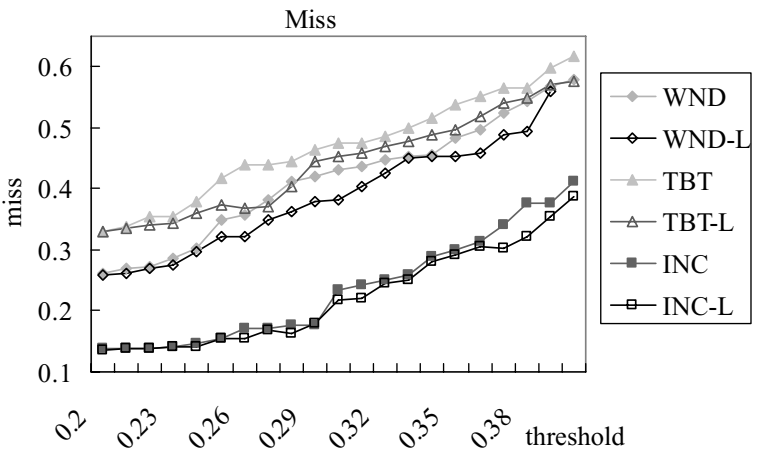

Fig. 12. The effects of thresholds on the miss rate. 


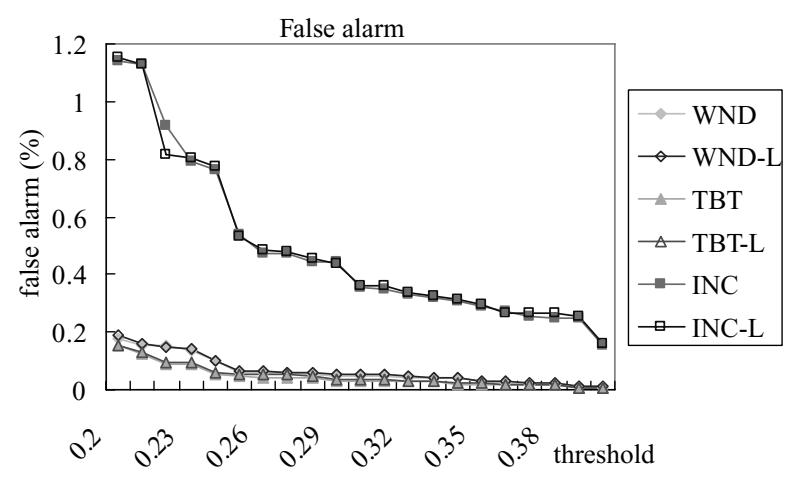

Fig. 13. The effects of thresholds on the false alarm rate.

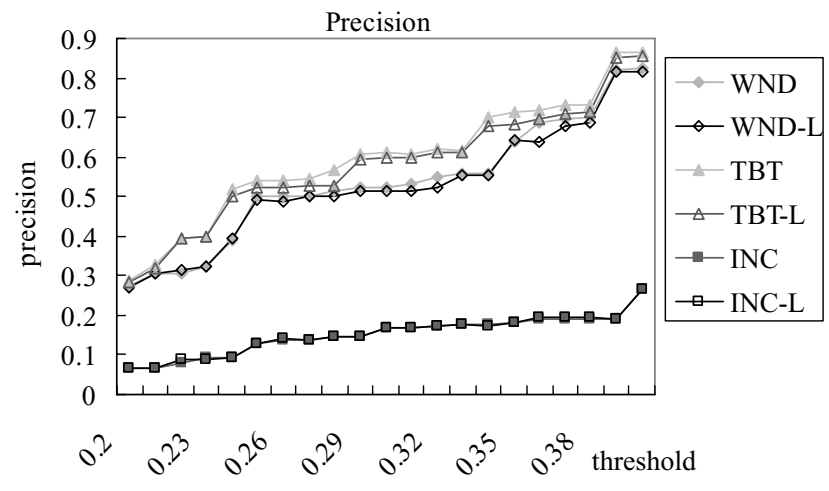

Fig. 14. The effects of thresholds on precision.

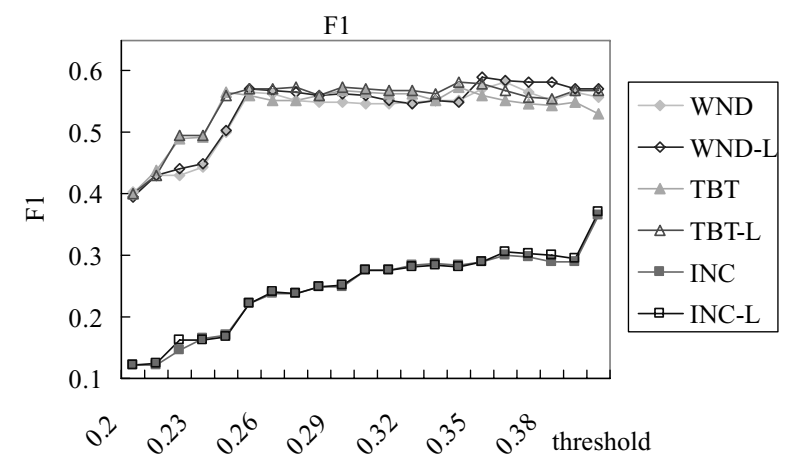

Fig. 15. The effects of thresholds on F1. 
Table VI. The One-Tailed Paired T-Test Analyses of LIPED Improvement

\begin{tabular}{|l|c|c|c|c|}
\hline & \multicolumn{2}{|c|}{ F1 } & \multicolumn{2}{c|}{ Normalized Cost } \\
\hline & $p$-value & Confidence & $p$-value & Confidence \\
\hline WND & $0.000219^{* * *}$ & $99.98 \%$ & $0.0000011^{* * *}$ & $99.99 \%$ \\
\hline TBT & $0.000204^{* * *}$ & $99.98 \%$ & $0.0000007^{* * *}$ & $99.99 \%$ \\
\hline INC & $0.026926^{* *}$ & $97.31 \%$ & $0.000477^{* * *}$ & $99.95 \%$ \\
\hline
\end{tabular}

Legend: confidence: confidence level.

et al. 1997] are closer to the coordinate origin than those of the original methods. This results in a better normalized cost performance, as shown in Figure 11. For each curve, we also designate the normalized cost score of the most balanced result. It is interesting to note from the above experiments that the magnitude of improvement in the normalized cost is greater than that for F1. F1 is the harmonic mean of precision and recall, and is often close to the minimum of the two metrics [Manning et al. 2008]. Generally, the F1 score rises only when the two metrics increase concurrently. On the other hand, normalized cost (which is the TDT official metric) is a weighted combination of misses and false alarms, and it is sensitive to the miss rate, as mentioned at the beginning of Section 5.3. In LIPED, we make use of the burst-and-diverse characteristic of news events to adjust the clustering threshold for the inclusion of diverse event documents. The inclusion of such documents leads to a better miss rate and thus improves the cost significantly. However, irrelevant documents may also be included, which would impact on the precision rate and the improvement in the F1 score.

Without considering the temporal information about events, the incremental clustering algorithm cannot discriminate context-similar events; hence it groups them in a single cluster incorrectly, even if the events are separated by a long time interval. This misclustering degrades the precision and the false alarm rate substantially, and makes detected events noisy. Setting a high threshold for the incremental clustering algorithm can alleviate such problems and produce a better F1 score. Both the time window method and the time-based threshold method use the temporal information about events and therefore perform better than the incremental clustering algorithm in terms of the F1 score. As the time-based threshold method adds a penalty value to the threshold for temporally remote documents, it usually can discriminate context-similar events and produces better false alarm and precision scores, as shown in Figures 13 and 14, respectively. However, the penalty will also exclude remote follow-up documents of a long-running event. In contrast, the time window method can usually cluster such documents; therefore, it yields better cost and miss (or recall) scores, as shown in Figures 11 and 12, respectively.

From the figures, we observe that the miss rate is inversely related to the false alarm rate no matter what method is employed. Raising the threshold improves the false alarm rate, but degrades the miss rate. Reducing the threshold can lead low miss and high clustering error rates. Similar tradeoffs also exist in the relationship between precision and recall. Even though the LIPED framework can not solve the tradeoff problems completely, it reduces the tradeoffs with adaptive thresholds and achieves a better detection performance. To sum 


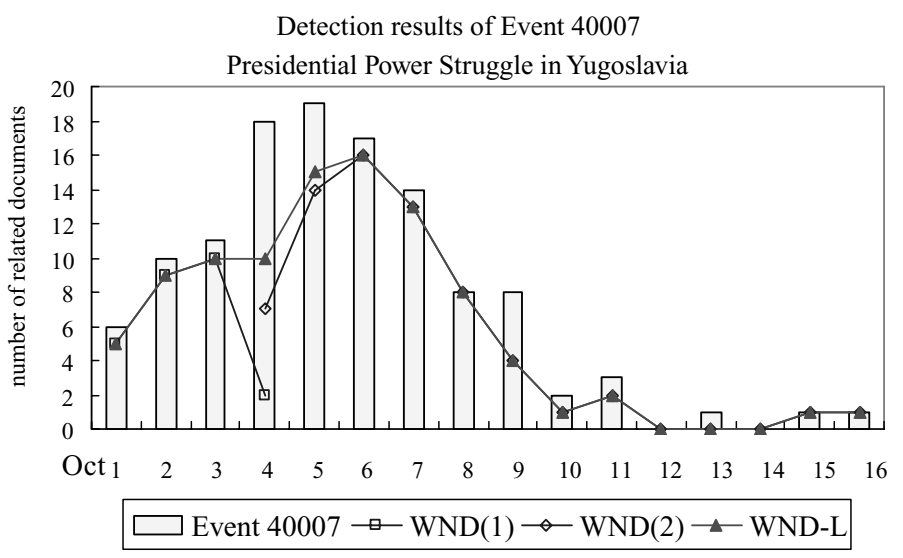

Fig. 16. Detection result of Event 40007 using the time window method.

up, LIPED improves the performance of existing algorithms in terms of the objective cost, miss rate, recall rate and $\mathrm{F} 1$ score, but not the false alarm and precision rates. It trades minor amounts of false alarm and precision for improvements in miss and recall, and for better objective cost and $\mathrm{F} 1$ performances.

\subsection{Example of Detecting Bursty Events}

In this section, we show the advantage of using LIPED with the time window method and the time-based threshold method on the following TDT4 events: 40007 Presidential Power Struggle in Yugoslavia and 40059 Attack on the USS Cole. As shown in Section 5.3.3, the precision of the incremental clustering algorithm is low. Thus, we do not consider its results because they are too noisy to provide clear illustrations. The life profiles employed in the following examples are of $\{\mathrm{C}-\mathrm{L}, \mathrm{L} 2, \mathrm{MT}\}$.

5.4.1 Example 1-Event 40007: Presidential Power Struggle in Yugoslavia. This event was related to the presidential election in Yugoslavia. In the first three days, the event focused primarily on incidents about strikes and protests because the incumbent refused to accept his defeat in the election. However, on day 4, the incumbent acknowledged the fact and the storyline immediately shifted from riots back to the election outcome. Figure 16 shows the result of the time window method with and without LIPED. The $x$-coordinate indicates the days of the event, and the $y$-coordinate records the number of related documents. The figure shows that the event was active in its early stage as there was an abundance of event documents. Then, the event exhibited the burst-anddiverse phenomenon on day 4 , but the time window method can not correctly detect the diverse documents using a rigid threshold (i.e., 0.36). It thus mistakenly breaks the whole event into fragmental clusters. For instance, the first cluster detected by the time window method (denoted as WND(1)) covers the documents related to the riots, while the second cluster (WND $(2))$ contains the remaining documents. By contrast, WND-L adaptively adjusts the clustering threshold (from 0.36, down to 0.33) according to the burst of the event, and 


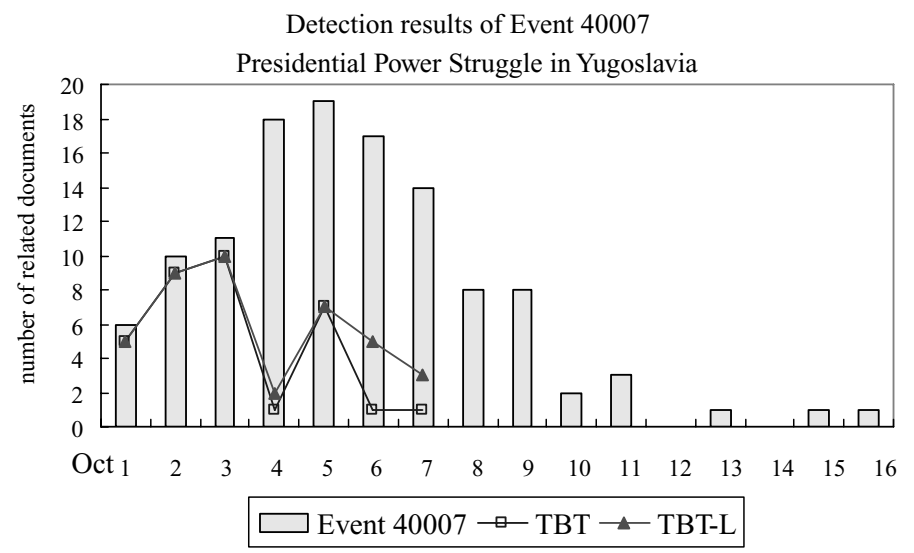

Fig. 17. Detection results for Event 40007 using the time-based threshold method.

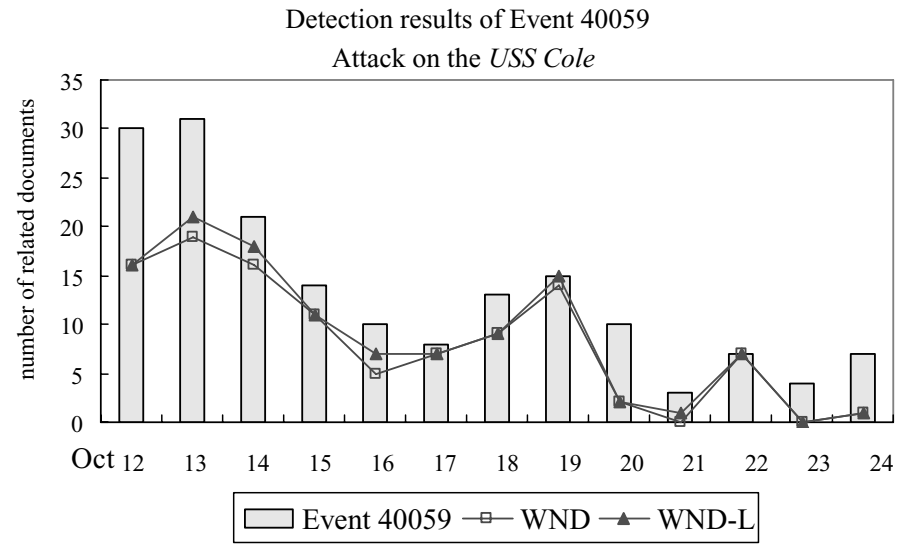

Fig. 18. Detection results for Event 40059 using the time window method.

includes more related documents without mistakenly identifying nonexisting events.

Figure 17 shows the detection results of the time-based threshold method with and without LIPED. As the method adds a penalty value to the clustering threshold for temporally remote documents, it tends to exclude follow-up documents of events, as shown in the figure. For instance, on days 6 and 7, the method can only cluster documents with similarities higher than 0.56 . Nevertheless, LIPED still includes more event documents by relaxing the threshold in response to the event burst.

5.4.2 Example 2-Event 40059: Attack on the USS Cole. Event 40059 was related to the attack on the American destroyer USS Cole in October 2000. As shown in Figure 18, the event was very active on the first day when there were 30 event documents. The documents mentioned many possibilities of who was responsible for the bombing and were very diverse. This type of event is challenging for LIPED because the burst-and-diverse phenomenon occurs too 


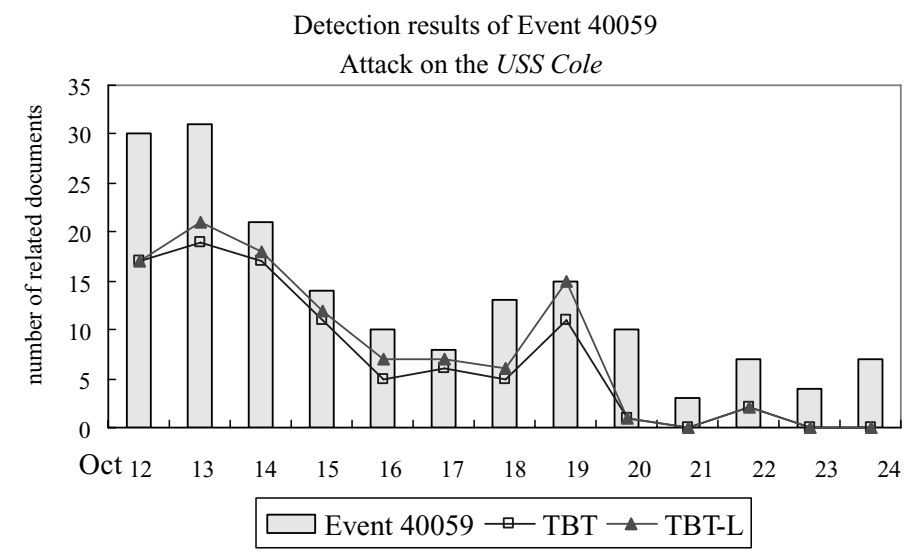

Fig. 19. Detection results for Event 40059 using the time-based threshold method.

early so there is insufficient historical information for threshold prediction. As a result, about half of the event documents for the first day are missed in clustering. Nevertheless, from the second day, LIPED immediately adjusts the clustering threshold according to the activeness status of the event. For instance, on October 13, LIPED adjusts the clustering thresholds of the time window method and the time-based threshold method from 0.36 to 0.34 and 0.34 to 0.32 , respectively, so that diverse event documents can be included.

\subsection{Time Complexity of LIPED}

In this section, we investigate the complexity of the LIPED framework. We calculate the execution time of the life profile learning phase and the event detection phase on an AMD AthlonTM 64 Processor 3200++ PC with the Windows XP Service Pack 2 operating system and a 2GB main memory.

5.5.1 Learning Complexity. We implement the life profile learning algorithm by using the famous Jahmm Java Package ${ }^{3}$ (version 0.4.1), which supports scaled calculations to avoid floating-point underflow. For each EMiteration, we calculate the negative log- likelihood between the training events and the learned life profiles. The log-likelihood indicates the closeness of the profiles and the training events. Obviously, the smaller the value, the better the life profiles will be. We found from the experiments that the life profiles produced by the complete-link strategy achieve superior negative log-likelihood performances. This is because the complete-link strategy is a conservative intercluster similarity strategy [Salton 1989], which makes the produced life profiles more cohesive than to those of the single-link strategy and the average-link strategy. Additionally, all three intercluster similarity strategies exhibit similar trends in terms of negative log-likelihood convergence. Figure 20 illustrates the impact of profile granularity on the convergence of the negative log-likelihood for the complete-link strategy. The figure shows that the learningt phase quickly

\footnotetext{
${ }^{3} \mathrm{http} / / /$ www.run.montefiore.ulg.ac.be/ francois/software/jahmm/ 


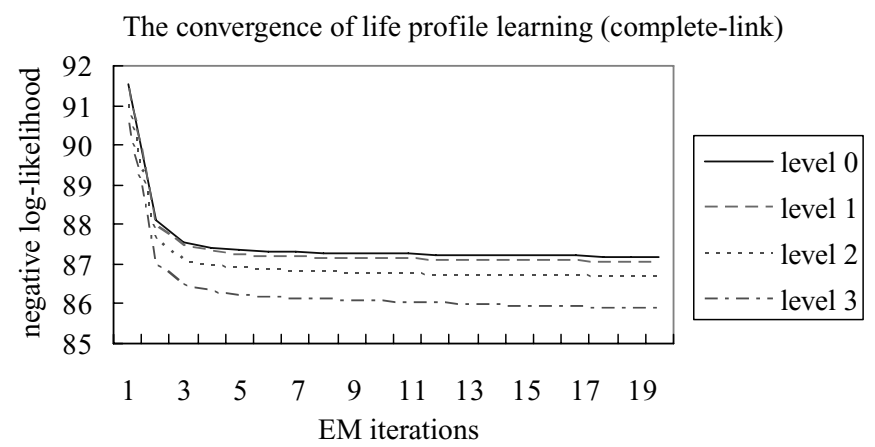

Fig. 20. The convergence of life profile learning using the complete-link strategy.

Table VII. The Execution Time of 20-Iterations of Life Profile Learning

\begin{tabular}{|l|c|c|c|}
\hline (sce.) & Single-link & Complete-link & Average-link \\
\hline level 0 & 14.6 & 14.6 & 14.6 \\
\hline level 1 & 14.8 & 14.8 & 14.7 \\
\hline level 2 & 14.9 & 15.1 & 15.1 \\
\hline level 3 & 14.9 & 15.7 & 15.5 \\
\hline
\end{tabular}

converges within a few EM-iterations because of the lengths of the training events. The life span of a TDT event is normally short, and short training events substantially reduce the complexity of profile learning. As shown in Table VII, the learning phase takes approximately 15 seconds to complete a 20 -iteration learning. The figure also shows that the negative log-likelihoods decrease with profile level increases. As mentioned previously, the levels determine the granularity of the life profiles. Deep profiles are specific and therefore produce superior negative log-likelihood values.

5.5.2 Event Detection Complexity. Figures 21, 22, and 23 show the execution times of the event detection methods with and without LIPED. The incremental clustering algorithm needs to examine all the already published documents in order to cluster a new document. Therefore, its execution time (1488 seconds) is long in comparison with that of the time window method and time-based threshold method where expired events are excluded from the detection process by employing the temporal information of clusters. LIPED certainly drags the event detection process and its execution time grows as the level of the employed life profiles increases. This is because deep levels contain many life profiles that LIPED needs to consider them all together to update the thresholds of events. Compared with the complete-link and average-link strategies, the single-link strategy generally produces less number of life profiles and therefore has fast event detection executions. According to Equations (9) and (10), the smoothed threshold strategy is more complex than the most likely threshold strategy; so its executions are longer than that of the most likely threshold strategy. 


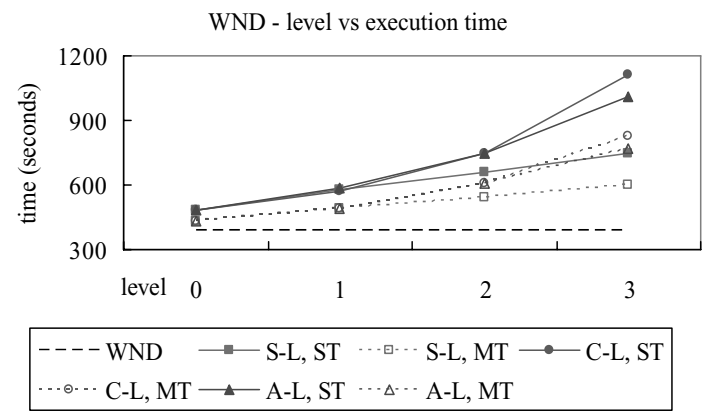

Fig. 21. The execution times of the time window method with and without LIPED.

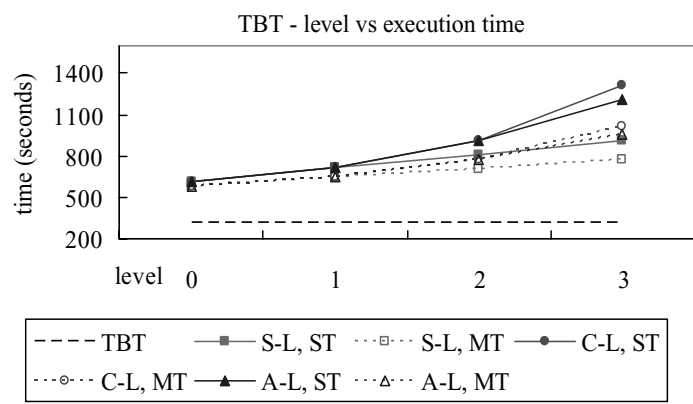

Fig. 22. The execution times of the time-based threshold method with and without LIPED.

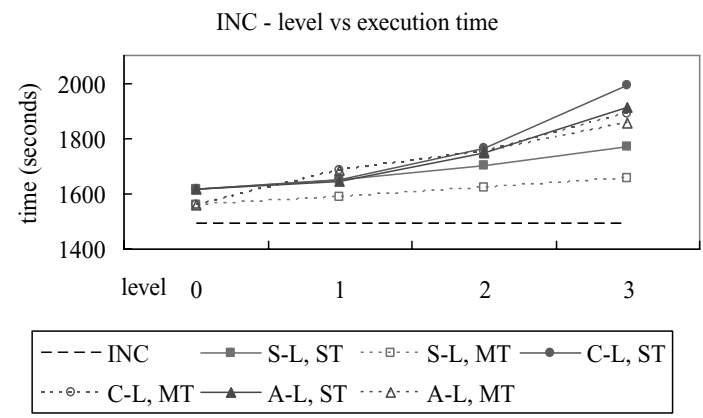

Fig. 23. The execution times of the incremental clustering algorithm with and without LIPED.

As the task of LIPED is to update the activeness status and threshold of each event once per time slot (i.e., every day), we divide the extra execution time of LIPED by the period of TDT4 and by the number of detected events, respectively, to investigate the overhead of LIPED in detail.

Figures 24 to 26 show that the daily overhead of LIPED is at most 8 seconds; thus, it is feasible for practical event detection systems. It is interesting that while the incremental clustering algorithm has the longest execution, its daily overhead is small. Basically, the daily overhead is proportional to the number of clusters because LIPED needs to update all the clusters at the end of a time slot. The incremental clustering algorithm tends to merge events so that a small 


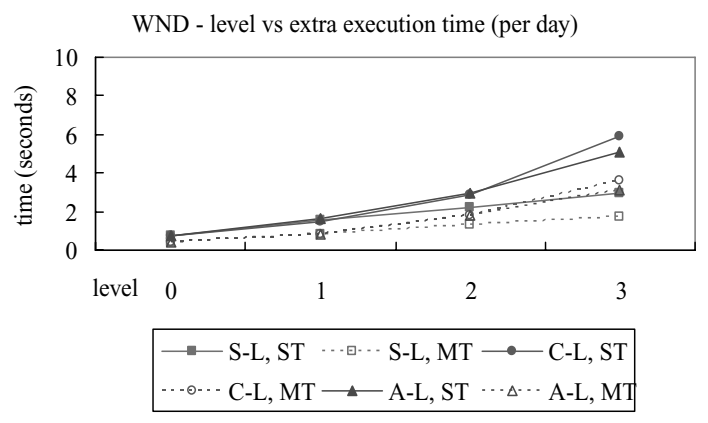

Fig. 24. The daily overhead of LIPED using the time window method.

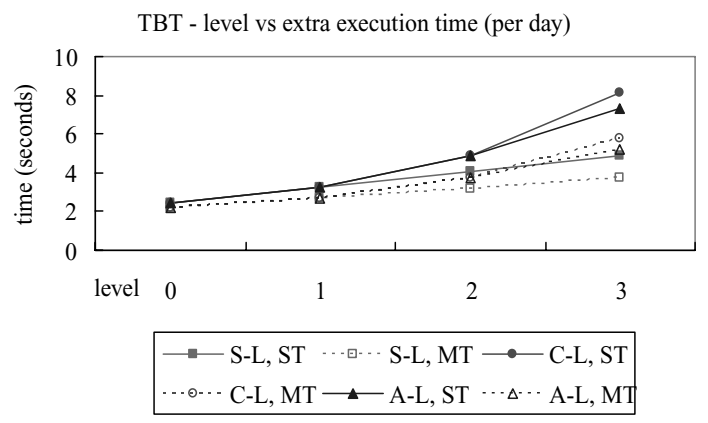

Fig. 25. The daily overhead of LIPED using the time-based threshold method.

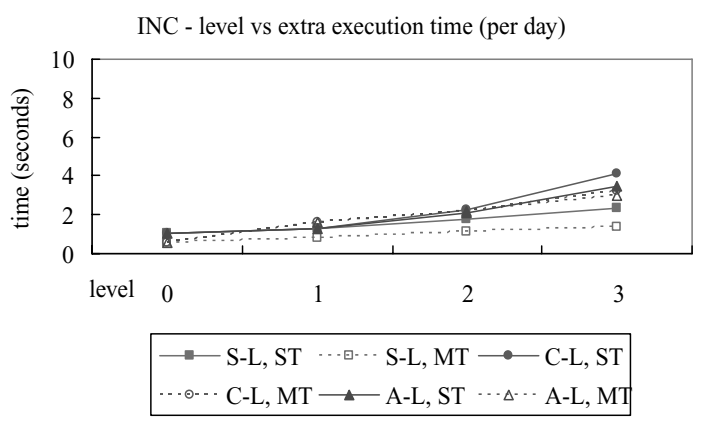

Fig. 26. The daily overhead of LIPED using the incremental clustering algorithm.

number of clusters reduce the daily overhead. Figures 27 to 29 show that the overhead for updating the activeness status and threshold is minor because the tasks can be performed within one millisecond. As mentioned in Section 4.2, the order of the update procedure is $O\left(N^{2} L\right)$. In practice, $N$ and $L$ are normally small numbers [Aizen et al. 2004; Kleinberg 2002] so that the update is a flash procedure. We found that different baseline methods lead to different update overheads, even if the set of employed life profiles is the same. Theoretically, the update overhead is irrelevant to the adoption of the baseline method, since LIPED only relies on the employed life profiles to update the status of an event. 


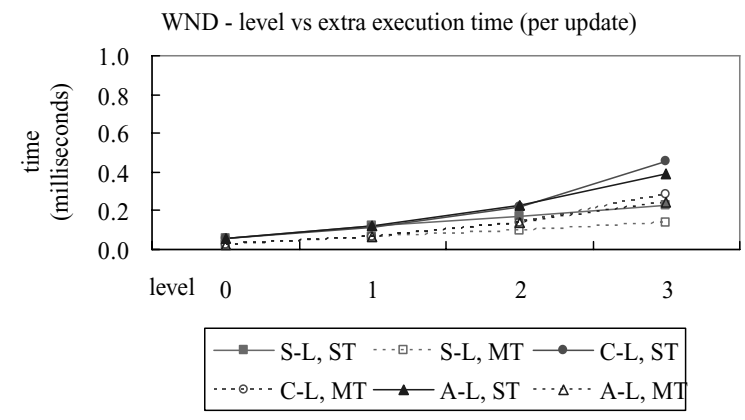

Fig. 27. The update overhead of LIPED using the time window method.

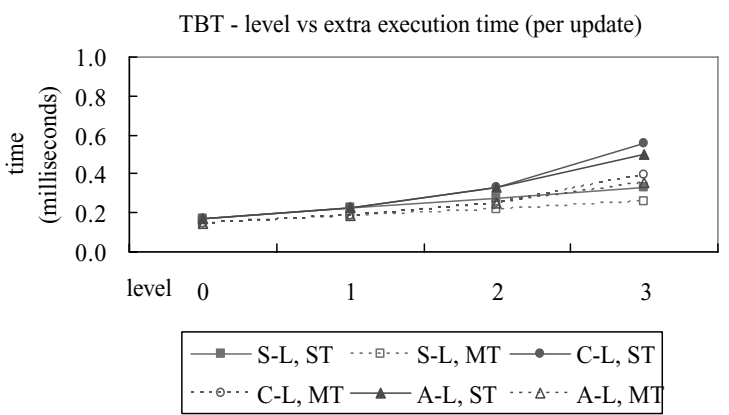

Fig. 28. The update overhead of LIPED using the time-based threshold method.

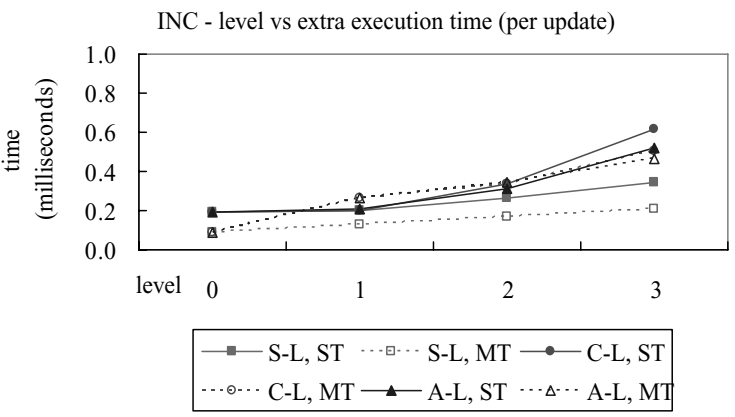

Fig. 29. The update overhead of LIPED using the incremental clustering algorithm.

We believe that the minor differences in update overheads are due to program implementation issues.

\section{CONCLUSIONS}

In this article, we have presented LIPED, which can be incorporated into existing event detection methods to improve their performance. We propose a generic framework to model the activeness trends of events using HMM-based life profiles. Also, learned life profiles are used to predict the activeness status of an event and adaptively adjust the clustering threshold during the event detection process. In evaluations using the official TDT4 corpus, existing methods 
achieve a $5.3 \%$ to $7.5 \%$ improvement in cost when incorporating LIPED. The improvements indicate that HMM-based life profiles can properly describe the activeness trends of news events.

The experiments reveal many interesting observations about event detection. First, most events in a document stream have the burst-and-diverse characteristic. Therefore, existing event detection algorithms can be enhanced by incorporating an adaptive threshold approach, such as LIPED. Second, the granularity of life profiles has a significant effect on LIPED performance. Life profiles that are too general (i.e., Aizen's method) fail to describe activeness trends adequately and result in poor detection performance. Third, setting appropriate thresholds for event detection is a challenging research issue as shown by the influence of threshold settings discussed in the previous section. Even though there is no reliable way to solve the problem, LIPED can be incorporated to improve performance.

The performance of LIPED depends on the quality of life profiles. As these profiles are learned from events with similar activeness developments, training data collection and life profile preparation are critical issues. Furthermore, as the outcome sequence of a training event can be viewed as a time series, life profile preparation is closely related to time series analysis. Therefore, stateof-the-art time series clustering methods may be used to enhance the LIPED system.

\section{ACKNOWLEDGMENTS}

The authors would like to thank the anonymous reviewers for their valuable comments and suggestions.

\section{REFERENCES}

AgGarwal, C. C. 2003. A Framework for diagnosing changes in evolving data streams. In Proceedings of the ACM SIGMOD International Conference on Management of Data. 575-586.

Aizen, J., Huttenlocher, D., Kleinberg, J., and Novak, A. 2004. Traffic-based feedback on the web. In Proc. Nat. Acad. Sci. 101, 525-5260.

Allan, J., Papka, R., and Lavrenko, V. 1998a. On-line new event detection and tracking. In Proceedings of the 21st Annual International ACM SIGIR Conference on Research and Development in Information Retrieval. 37-45.

Allan, J., Carbonell, J., Doddington, G., Yamron, J., and Yang, Y. 1998b. Topic detection and tracking pilot study: final report. In Proceedings of the DARPA Broadcast News Transcription and Understanding Workshop. 194-218.

Allan, J., Lavrenko, V., Frey, D., and Khandelwal, V. 2000. Proceeding of the TDT Workshop. Baeza-Yates, R. and Ribeiro-Neto, B. 1999. Modern Information Retrieval. Addison-Wesley.

Barlas, Y. AND KANAR, K. 1999. A dynamic pattern-oriented test for model validation. In Proceedings of 4th Systems Science European Congress. 269-286.

Baum, L. E., Petrie, T., Soules, G., And Weiss, N. 1970. A maximization technique occurring in the statistical analysis of probabilistic functions of Markov chains. Ann. Math. Statist. 41, 164-171.

Chen, C. C., Chen, Y. T., Sun Y., And Chen, M. C. 2003. Life cycle modeling of news events using aging theory. In Proceedings of the 14th European Conference on Machine Learning. 47-59.

Chen, C. C., Chen, M. C., And Chen, M. S. 2005. LIPED: HMM-based life profiles for adaptive event detection. In Proceedings of the 11th ACM SIGKDD International Conference on Knowledge Discovery and Data Mining. 556-561.

Chou, T. C. And Chen, M. C. 2008. Using incremental PLSI for threshold-resilient online event analysis. IEEE Trans. Know. Data Eng. 20, 3, 289-299. 
Cormen, T. H., Leiserson, C. E., Rivest, R. L., And Stein, C. 2001. Introduction to Algorithms. MiT Press.

Dempster, A. P., Laird, N. M., and Rubin, D. B. 1977. Maximum likelihood from incomplete data via the EM algorithm. J. Royal Statist. Soc. Series B 39, 1-38.

Donjerkovic, D. and Ramakrishnan, R. 2000. Dynamic histograms: capturing evolving data sets. In Proceedings of the 16th International Conference on Data Engineering. 86.

Fiscus, G. J. AND DodDington, G. 2002. Topic detection and tracking evaluation overview. In Topic Detection and Tracking: Event-Based Information Organization. Kluwer Academic Press, 1730.

Frakes, W. B. And Baeza-Yates, R. 1992. Information Retrieval: Data Structures and Algorithms. Prentice Hall.

Franz, M., McCarley, J. S., Ward, T., AND Zhu, W. J. 2001. Unsupervised and supervised clustering for topic tracking. In Proceedings of the 24th Annual International ACM SIGIR Conference on Research and Development in Information Retrieval. 310-317.

Ganti, V., Gehrke, J., AND RAmakrishnan, R. 1999. A framework for measuring changes in data characteristics. In Proceedings of the Eighteenth ACM SIGMOD-SIGACT-SIGART Symposium on Principles of Database Systems. 126-137.

Ghahramani, S. 2000. Fundamentals of Probability. Prentice Hall.

Hatzivassiloglou, V., Gravano, L., And Maganti, A. 2000. An investigation of linguistic features and clustering algorithms for topical document clustering. In Proceedings of the 23rd Annual International ACM SIGIR Conference on Research and Development in Information Retrieval. 224-231.

KLEINBERG, J. 2002. Bursty and hierarchical structure in streams. In Proceedings of the 8th ACM SIGKDD International Conference on Knowledge Discovery and Data Mining. 91-101.

LEUSKI, A. AND Allan, J. 2002. Improving realism of topic tracking evaluation. In Proceedings of the 25th Annual International ACM SIGIR Conference on Research and Development in Information Retrieval. 89-96.

LI, Z., WANG, B., LI, M., AND MA, W-Y. 2005. A probabilistic model for retrospective news event detection. In Proceedings of the 28th Annual International ACM SIGIR Conference on Research and Development in Information Retrieval. 106-113.

Makkonen, J., Ahonen-Myka, H., and Salmenkivi, M. 2004. Simple semantics in topic detection and tracking. Information Retrieval. 7, 3-4, 347-368.

Manmatha, R., Feng, A., and Allan, J. 2002. A critical examination of TDT's cost function. In Proceedings of the 25th Annual International ACM SIGIR Conference on Research and Development in Information Retrieval. 403-404.

Manning, C. D., Raghavan, P., And Schutze, H. 2008. Introduction to Information Retrieval. Cambridge University Press.

Markov, A. A. 1913. An example of statistical investigation in the text of 'Eugene Onyegin' illustrating coupling of 'tests' in chains. In Proc. Acad. Sci. 7, 153-162.

Martin, A., Doddington, G., Kamm, T., Ordowski, M., And Przybocki, M. 1997. The DET curve in assessment of detection task performance. In Proc. EuroSpeech, 4, 1985-1898.

MitcheLl, T. M. 1997. Machine Learning. McGraw-Hall.

Myers, C., Rabiner, L. R., and Rosenberg, A. E. 1980. Performance tradeoffs in dynamic time warping algorithms for isolated word recognition. IEEE Trans. Acoust. Speech Signal Proc. 28, 6, 623-635.

PAPKA, R. 1999. P.h.D thesis, Department of Computer Science, University of Massachusetts.

RABINER, L. R. 1989. A tutorial on hidden Markov models and selected applications in speech recognition. In Proc. IEEE 77, 2, 257-286.

RIJSBERGEN, C. J. V. 1979. Information Retrieval, 2nd. Butterworths, London.

Rocchio, J. J. 1971. Relevance feedback in information retrieval, In The SMART Retrieval System, Prentice Hall, 313-323.

Salton, G. 1989. Automatic Text Processing: the Transformation, Analysis, and Retrieval of Information by Computer. Addison-Wesley.

Silverman, B. 1986. Density Estimation for Statistics and Data Analysis. Chapman and Hall.

ViterBi, A. J. 1967. Error bounds for convolutional codes and an asymptotically optimum decoding algorithm. IEEE Trans. Inform. Theory IT-13, 1260-1269.

ACM Transactions on Information Systems, Vol. 27, No. 2, Article 9, Publication date: February 2009. 
Yang, Y., Pierce, T., ANd Carbonell, J. 1998. A study on retrospective and on-line event detection. In Proceedings of the 21st Annual International ACM SIGIR Conference on Research and Development in Information Retrieval, 28-36.

Zhang, J., Ghahramani, Z., AND YANG, Y. 2004. A probabilistic model for online document clustering with application to novelty detection. In Proceedings of the Conference on Neural Information Processing System. 1617-1624.

Received September 2006; revised October 2007; accepted June 2008 